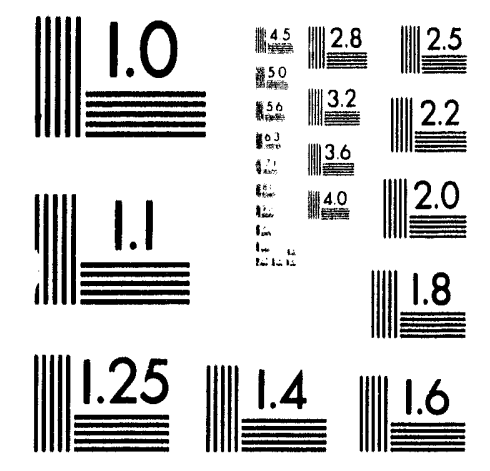



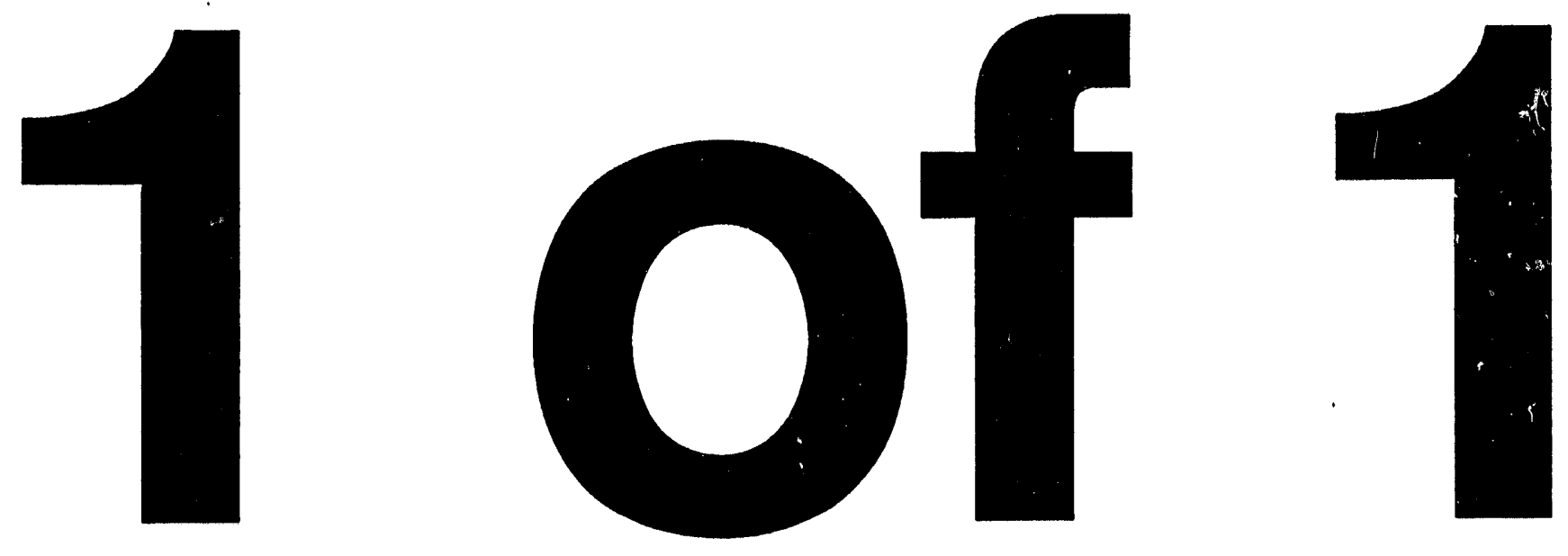
DOE/ER/40452-9

\title{
RESEARCH AND DEVELOPMENT OF A HELIUM-4 BASED SOLAR NEUTRINO DETECTOR
}

\section{PROGRESS REPORT}

for Period November 1, 1991 - April 30, 1993

\author{
R. E. Lanou, H. J. Maris, G. M. Seidel \\ Department of Physics \\ Brown University \\ Providence, RI 02912
}

May 1993

Prepared for:

U.S. DEPARTMENT OF ENERGY

Grant No. DE-FG02-88ER40452

\section{MASTRR}

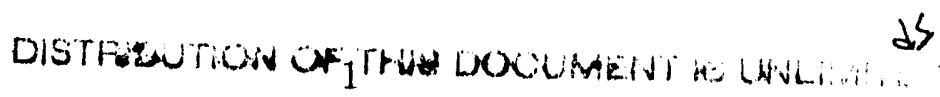




\section{NOTICE}

This report was prepared as an account of work sponsored by the United States Government. Neither the United States, nor the Department of Energy, nor any of their employees, nor any of their contractors, subcontractors, or their employees, makes any warranty, expressed or implied, or assumes any legal liability or responsibility for the accuracy, completeness, or usefulness of any information, apparatus, product or process disclosed or represents that its use would not infringe privately-owned rights. 


\section{TABLE OF CONTENTS}

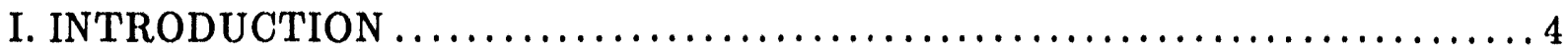

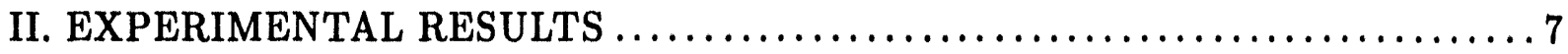

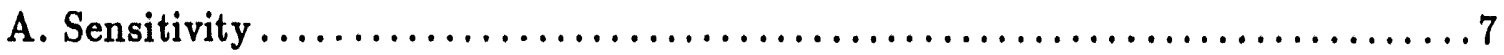

B. Heat Capacity of Wafer/Calorimeters $\ldots \ldots \ldots \ldots \ldots \ldots \ldots \ldots \ldots \ldots \ldots \ldots, \ldots$

C. Coincidence Measurements $\ldots \ldots \ldots \ldots \ldots \ldots \ldots \ldots \ldots \ldots \ldots \ldots \ldots \ldots \ldots$

D. Spectrum of Alpha Particles in Helium............................10

E. Quantum Evaporation: Angular Dependence and Efficiency $\ldots \ldots \ldots \ldots \ldots 11$

III. BACKGROUND STUDIES FOR A HELIUM DETECTOR................14

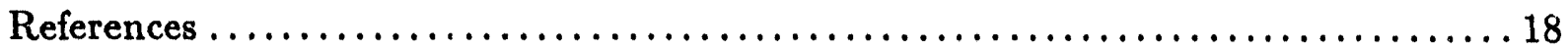

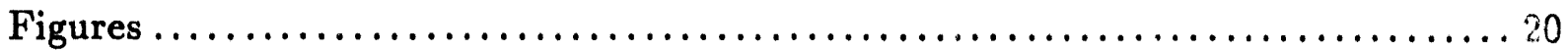

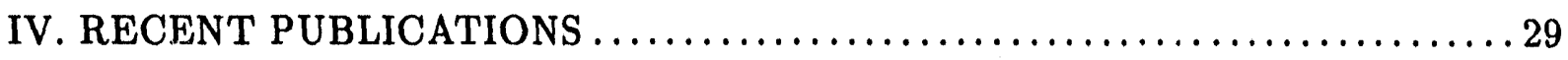

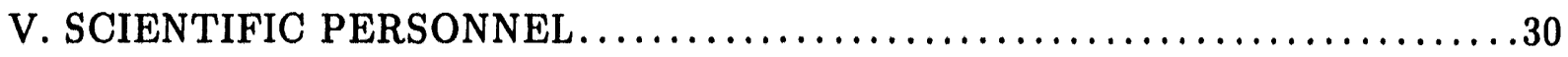

APPENDIX A. PRINCIPLES OF THE SUPERFLUID HELIUM DETECTOR ... 31 


\section{INTRODUCTION}

We report here on a research project to develop a method for the measurement of the spectrum of very low energy neutrinos from the sun - the neutrinos generated by the $\mathrm{p}-\mathrm{p}$ and ${ }^{7} \mathrm{Be}$ reactions. The detection of these neutrinos and the measurement of their energies is important for several reasons. The flux expected for the p-p neutrinos is highly constrained by the optical luminosity and depends only slightly on the astrophysical model of the sun. The ${ }^{7} \mathrm{Be}$ neutrinos constitute a line spectrum and have a dependence on solar temperature quite different from that of the $p-p$ as well as from that of the high energy ${ }^{8} \mathrm{~B}$ neutrinos. Taken together, measurements of the total (i.e., all neutrino types) fluxes and spectra from all three major reactions would provide all the data essential to a full resolution of the "solar neutrino problem" (SNP); and if it is established that the cause of the problem is due to the properties of the neutrino, this would represent the first evidence for new physics beyond the "standard model" of nuclear and particle physics.

The original results from the continuing, pioneering and elegant experiments (the radiochemical experiments of Homestake, SAGE and GALLEX; the water Cerenkov experiments Karniokande II \& III) have defined the SNP. The new results from these same experiments have increased our knowledge of the problem and confirmed its magnitude. Additionally, there has been a convergence to a common set of predicted rates of electron-neutrinos by the various groups who calculate the "standard solar models" (SSM) - it would now appear that there is an agreed upon standard model to within a few percent [1]. Thus the comparison between the new results and the rates to be expected takes on greater significance. These new comparisons are as follows: a) the Homestake experiment [2] $(\mathrm{Cl} \rightarrow \mathrm{Ar})$ is mainly sensitive to the ${ }^{8} \mathrm{~B}$ and ${ }^{7} \mathrm{Be}$ and sees 0.26 \pm 0.04 of the SSM ( $a \sim 6$ standard deviation effect), b) the combined Kamiokande II and III [3] (water Cerenkov) result is $0.50 \pm 0.07$ of the SSM (a $\sim 3$ standard deviation effect), however, it is sensitive to only the ${ }^{8} \mathrm{~B}$ neutrinos, c) the SAGE [4] and the GALLEX. [5] experiments $(\mathrm{Ga} \rightarrow \mathrm{Ge})$ are sensitive to $\mathrm{p}-\mathrm{p}$ neutrinos giving a cumulative $0.44 \pm 0.16$ for $S A G E$ and $0.63 \pm 0.16$ for GALLEX in comparison to SSM. Some authors (see Bludman et al [6]) have combined the SAGE and GALLEX results to estimate a $0.54 \pm 0.13$ of SSM for the combination and suggest it is a 3.7 standard deviation effect.

There is now a growing consensus [6] that these experiments and their comparison to the now reasonably firmly constrained SSM make it more difficult to make a selfconsistent interpretation in terms of straightforward adjustment of the parameters in the SSM. Rather, it is generallly expected that the correct explanation of the results

will involve the introduction of new physics regarding neutrinos. There is, however, 
no understanding of what these neutrino properties might be beyond, possibly, leptonnumber non-conservation and a mass-squared difference among the three neutrino types (e.g., MSW matter vs. vacuum oscillations; Bludman et al vs. Barger et al [6]).

For some time, well before these exciting new developments, it has been realized that a full understanding of the SNP would require that two things be experimentally determined: a) the full neutrino beta decay energy spectrum from the sun must be measured and b) the full flux - not just that of electron-type neutrinos - be measured for the three major reactions in the sun. This is as true today as it was earlier, and the next generation of detectors now under construction (SNO and Superkamiokande) will be making a major advance in these goals, particularly in their first significant measurement of neutral current processes, which will test whether the neutrino-types $\nu_{\mu}$ and $\nu_{\tau}$ exist in the solar flux. If the European experiment Borexino is constructed, then there will be real-time measurement of the flux from the ${ }^{7} \mathrm{Be}$ reaction, providing further essential information by broadening the spectrum sampled.

There remains, then, the important challenge of measuring the neutrino flux from the primary $p-p$ reaction in the sun in real time, with sensitivity to the neutral current and with energy resolution.

Such a measurement is important for several reasons, among them the fact that it is the least dependent on solar models. Also, the ratio of the $\nu_{e}$ flux to the overall neutrino flux must be known for all three major reactions before an over-determined solution is established. Should the solution to the SNP be related with the neutrino mass, then the ambiguities in masses and mixing parameters are likely to require as many details of each of the three reactions as can be measured. Because relative flux comparisons among the three reactions are key ingredients in seeking an understanding, it is also valuable to measure more than one reaction in the same experiment so that systematic effects can be minimized.

It is a challenge to measure the $\mathrm{p}-\mathrm{p}$ reaction because, although it has a copious flux $\left(6 \times 10^{10} \mathrm{~cm}^{-2} \mathrm{sec}^{-1}\right.$ at the earth $)$, the energies are very low $(<420 \mathrm{keV})$. The gallium radiochemical experiments have established beautifully the $\nu_{e}$ flux for p-p solar neutrinos. To go further and to measure the flux of all neutrinos and to do it in real time is the next necessary step. It implies a need for exceptional radioactive purity of target and control of backgrounds. The techniques to do this have not existed, and it is to achieve this goal that we have been developing the superfluid helium detection method. If successful it will allow the simultaneous measurement of these quantities for both the $p-p$ and ${ }^{7}$ Be reactions in one experiment.

The principles of our novel method to use superfluid helium as the neutrino target 
and as the medium for energy cieposition have been described in detail in several of our publications [7] and for the reader's convenience are summarized in Appendix A. The method capitalizes on two remarkable properties of helium. The first is that helium at low temperature is the purest known material, and since an almost complete absence of radioactivity is essential for the large amount of target material needed( $\sim 10$ tons), it is the ideal candidate on that score. The second property is that the energy deposited in the helium is carried out of the large volume by elementary excitations of the liquid (phonons and rotons) which can produce quantum evaporation of helium atoms at a free surface. These atoms are easily collected and detected on low mass wafers (e.g., silicon) by a calorimetric method. Energy is measured by determining the transient temperature rise the wafers.

In our previous Progress Report we reported the completion of construction of the cryogenic apparatus and on the results of experiments in which $\alpha$ particles and electrons have been detected. We also reported follow-on experiments that begin to investigate some of the details of the technique needed in demonstrating the feasibility of a full-scale neutrino detector. In this Progress Report we detail our new experiments, including the intriguing discovery of effects which may lead to determining the original direction of the recoiling particle. 


\section{EXPERIMENTAL RESULTS}

Progress has been made on several fronts in this ten month period since our last Report (DOE/ER/40452-7). We briefly touch on several areas and emphasize a potentially important discovery concerning the generation of rotons in liquid helium by charged particles, a discovery which holds promise of providing a means of the determining the direction of the particle track.

\section{A. Sensitivity}

Because the initial goal of our research program has primarily been to establish that our understanding of the basic processes associated with particle detection in liquid helium is correct, we selected as the thermistor for the wafer/calorimeters the readily available neutron transmutation doped (NTD) germanium [8]. This type of high impedance device when used with commercial electronics was a quick means of getting "on the air". The system has served admirably in exploring the quantum evaporation using 5.5 $\mathrm{MeV} \alpha$ particles. However, NTD germanium is unlikely to be the sensor of choice for a full-scale solar neutrino detector. Thin film technology is much more compatible with the fabrication requirements of a large multi-wafer system than is a technology based on discrete elements that must be glued the wafers. Also, evaporated superconducting transition-edge bolometers [9] presently possess higher sensitivities than NTD Ge and have much more potential for further improvement.

We have begun work on the development of superconducting transition-edge thermometers for sensing the temperature pulses on the wafers. The cryostat has been instrumented with three channels of dc SQUID electronics. Transition-edge thermometers are typically low impedance devices ideally matched to SQUID's. To date we have measured the properties of a thin film of tungsten, which in bulk form has a transition and $15 \mathrm{mK}$, and an epitaxially grown crystalline film of beryllium, the bulk transition being at $23 \mathrm{mK}$. We have discovered that these elemental superconductors as thin films have drastically different properties than the bulk. In this regard they appear similar to aluminum, the thin film properties of which, although studied extensively, are not well understood. Rather than get involved in a potentially lengthy research project to develop these materials to the point that they are useful to us, we shall shift our attention to alloys that have transitions in the $20 \mathrm{mK}$ temperature region and are known to make suitable thermometers. For example, the Munich group [9] has demonstrated a sensitivity of $300 \mathrm{eV}$ with a $31 \mathrm{gram}$ crystal of sapphire using a gold-iridium alloy at 
$40 \mathrm{mK}$. This thermometer, which is two orders of magnitude more sensitive than our present devices, is of sufficient quality to make a large-scale neutrino detector feasible.

\section{B. Heat Capacity of Wafer/Calorimeters}

The sensitivity to small energy inputs depends not only on the measurement of the temperature but $\mathrm{cn}$ the heat capncity of the calorimctcr as well; $\delta T=\delta E / C$. Using an $\alpha$ particle incident directly on a wafer as a known energy input, we have determined the heat capacity of several wafers of silicon and of sapphire, dielectric crystals with high Debye temperatures. The measured heat capacity for one silicon wafer is shown in Fig. 1 where $\mathrm{C} / \mathrm{T}$ is plotted against $\mathrm{T}^{2}$ between $20 \mathrm{mK}$ and $150 \mathrm{mK}$ [10]. The heat capacity of all of our wafers can be fit by the relation

$$
C=a T+b T^{3},
$$

and the results for four different wafers are summarized in the accompanying table.

\begin{tabular}{|l|l|l|l|l|}
\hline Material & Size & $\mathrm{a}\left(\mathrm{J} / \mathrm{K}^{2}\right)$ & $\mathrm{b}\left(\mathrm{J} / \mathrm{K}^{4}\right)$ & Debye $\left(\mathrm{J} / \mathrm{K}^{4}\right)$ \\
\hline silicon & $9.2 \mathrm{~cm}^{2} \times 0.3 \mathrm{~mm}$ & $3.0 \times 10^{-9}$ & $2.3 \times 10^{-7}$ & $2.1 \times 10^{-7}$ \\
\hline silicon & $1.2 \mathrm{~cm}^{2} \times 0.3 \mathrm{~mm}$ & $1.4 \times 10^{-9}$ & $2.2 \times 10^{-8}$ & $2.3 \times 10^{-8}$ \\
\hline sapphire & $20 \mathrm{~cm}^{2} \times 0.3 \mathrm{~mm}$ & $2.8 \times 10^{-9}$ & $2.5 \times 10^{-7}$ & $2.9 \times 10^{-7}$ \\
\hline sapphire & $45 \mathrm{~cm}^{2} \times 0.6 \mathrm{~mm}$ & $2.0 \times 10^{-9}$ & $1.5 \times 10^{-6}$ & $1.3 \times 10^{-6}$ \\
\hline
\end{tabular}

The last column of the table indicates the expected magnitude of the Debye $\mathrm{T}^{3}$ term in the heat capacity for the various samples. It is clear that the measured $\mathrm{T}^{3}$ component agrees with the theoretical value for both silicon and sapphire, but in addition there exists a large linear term that dominates the heat capacity in the low temperature region. The presence of this additional heat capacity decreases the sensitivity of our calorimeters by a factor of 10 , and its origin is a subject of concern and ongoing research.

A heat capacity linear in $\mathrm{T}$ is not uncommon in dielectrics at low temperatures and is usually associated with two-level systems. Two-level systems could be located in the bulk of the wafers, in a layer of disordered material at the surface, or possibly in the epoxy adhesive used to attach the Ge thermistor. We continue to investigate the source of this added heat capacity so as to be able eventually to reduce it and increase our sensitivity substantially.

It is possible to study the time dependence of the thermal response of the wafer using $\alpha$ particles incident directly on it. At low temperatures it is found that the pulse 
height of the thermal signal varies by $\sim 15 \%$ for a $0.3 \mathrm{~mm}$ thick, sapphire wafer of $20 \mathrm{~cm}^{2}$ area, as shown in Fig. 2. This is a far broader distribution than the energy spectrum of the incident $5.5 \mathrm{MeV} \alpha$ particles from ${ }^{241} \mathrm{Am}$. However, if the $\alpha$ particles are collimated so that they all strike the wafer within a $\sim 0.2 \mathrm{~cm}^{2}$ area, the measured distribution is narrowed to $\sim 1 \%$ as shown in Fig. 3. The dependence of the amplitude of the thermal signal to the location of the energy deposition indicates that the rate at which the wafer achieves internal thermal equilibrium is only slightly faster than the rate at which it comes to equilibrium with its heat sink. Nonetheless, the variations in pulse size are small and certainly acceptable. In a neutrino detector the energy input to the wafer from the adsorption of helium atoms will be spread reasonably uniformly over the surface and the thermal coupling to the reservoir can be made weaker. The thermal signal will directly measure the energy input.

\section{Coincidence Measurements}

As discussed in our last Progress Report, we have completed a re-configuration of the interior of our test cell. The interior diameter of the cell has been increased to 15 $\mathrm{cm}$ so that 3 liters of helium are contained in the volume below the wafers. We now have provision for placing a number of wafers in the cell and reading as many as 4 of them simultaneously. Also tiny superconducting stepping motors are mounted inside the cell to permit studies with a movable radioactive source as well as with other test pieces [11]. These modifications have given us the ability to study 1) discrimination of signals from background by coincidence measurements, 2) angular dependence of quantum evaporation and 3 ) reflection of rotons from solid surfaces.

A schematic diagram of the test cell, with two wafers illustrated, is shown in Fig. 4. An ${ }^{241} \mathrm{Am} \alpha$ source is mounted on the movable arm of a stepping motor. The results discussed in this section were obtained with the source in a fixed position symmetrically below two wafers and $5 \mathrm{~cm}$ beneath the liquid surface. (The interesting new results derived from measurements with the movable source are discussed in Section II E.) In Fig. 5 is illustrated a typical $\alpha$ event depositing roughly $200 \mathrm{keV}$ to one of the sapphire wafers at $30 \mathrm{mK}$, a coincident signal having been recorded by the second wafer. The signal-to-noise is a very comfortable 10 to 1 . The noise, which originates primarily from the preamplifier in the detection system, is equivalent to $20 \mathrm{keV}$ in this particular experiment.

The pulse height spectrum of energy depositions as recorded by one of the wafers (in coincidence) is shown in Fig. 6. That this spectrum is due entirely to $\alpha$ particles, 
except for the noise peak at $\sim 20 \mathrm{keV}$, is confirmed by moving the source away from the wafers and into a shielded enclosure on the side of the cell. The coincident signals are no longer present except for the noise-generated component between 10 and $30 \mathrm{keV}$. The data in Fig. 6 is raw in the sense that no subtraction for the noise signal has been applied nor has any real-time or off-line filtering or pulse shaping been utilized. What the coincidence measur ent guarantees is that the signals are generated by events in the liquid. Heat pulses from backgzound $\gamma^{\prime}$ s interacting in a wafer are eliminated from the data sample. The size of the signals produced by $\gamma^{\prime} s$ that interact with the liquid helium is below our energy threshold.

\section{Spectrum from Alpha Particles in Helium}

Of particular interest is the unusual nature of the spectrum of energy depositions from the $\alpha$ 's stopped in the helium [12], as shown in Fig. 6. The spectrum consists of a peak and a broad shoulder on the high energy side. The shape of the spectrum has nothing to do with any spread in energies of the $\alpha$ 's from the source, the width of which, as measured by a surface-barrier detector, is $\sim 20 \mathrm{eV}$. Nor is the shape or distribution influenced significantly by statistical effects. To deposit 100 ke $V^{\prime}$ of energy onto a wafer requires the adsorption of $10^{7}$ helium atoms. We believe that ther are two likely contributions to the unusual shape of the spectrum, 1) the presence of a substrate on which the source is deposited and 2) a dependence of the energy of the detected signal on the direction of motion of the $\alpha$ particle. We have begun instrumenting experiments to test these hypotheses. The possible influence of the substrate is discussed in the following paragraph, while the dependence of signal on track direction is considered towards the end of the next section. We discuss first measurements of the angular variation of the detected signal, which provide supporting evidence for the dependence on track direction.

The ${ }^{241} \mathrm{Am}$ source is imbedded within an evaporated stainless steel matrix approximately $0.1 \mu \mathrm{m}$ thick on a glass substrate. While the range of a $5.5 \mathrm{MeV} \alpha$ particle is $\sim 200 \mu \mathrm{m}$ in liquid helium, it is only $\sim 20 \mu \mathrm{m}$ in glass. The energy deposited by an $\alpha$ propagating in the glass is very close to the surface. We believe that a considerable fraction of the thermal excitations created in the glass substrate will travel to the surface in contact with the helium and produce sufficient rotons in the liquid that a signal is recorded on the wafers. Evidence in support of this mechanism is that the rate of events in the measured spectrum shown in Fig. 6 is consistent with the known activity of the source and the rate at which $\alpha$ particles are emitted into the full $4 \pi$ solid angle, rather 
than into the $2 \pi$ angle subtended by the liquid. To investigate this matter further our next measurements will be conducted with an especially prepared, freely suspended, 0.1 $\mu \mathrm{m}$ thick, substrate-free source.

\section{E. Quantum Evaporation: Angular Dependence and Efficiency}

In investigating the physical processes important in the furctioning of a detector of solar neutrinos, we have studied the dependence of quantum evaporation on the incident angle of the excitations at the surface using both $\alpha$ particles and a resistance heater as sources [13]. From these measurements using the $\alpha$ source we can make an estimate of the efficiency of evaporation.

The two sources are located $1.5 \mathrm{~cm}$ below the liquid surface and can be moved laterally through a distance of $12 \mathrm{~cm}$ by a superconducting stepping motor. The quantum evaporation signal measured by $1 \mathrm{~cm}^{2}$ wafer/calorimeter for a $1 \mu \mathrm{J}$ heat pulse into the heater is shown in Fig. 7. The horizontal axis represents the lateral distance of the heater from the center of the wafer. For a given size of heat pulse and distance, the amplitude of the signal is constant to within a few percent. As expected the maximum amplitude as a function of position occurs with the source directly below the wafer, and the signal decreases rapidly with angle.

Wyatt [14] has shown, using collimated bo ams of rotons and phonons, that the component of momentum parallel to the surface and the energy are conserved in the evaporation of an atom by an excitation in the liquid. These conservation conditions result in a critical angle beyond which quantum evaporation cannot occur, not unlike the critical angle of total internal reflection in optics. The critical angle varies with momentum as shown in Fig. 9, from less than $20^{\circ}$ near the roton minimum to much larger values at higher energies. Based on these conditions we have constructed a Monte Carlo simulation to analyze the data more quantitatively. In this simulation excitations are generated with momenta in random directions and energies related to the momenta through the dispersion relation for helium. Excitations striking the surface are assumed to produce evaporation with unit probability provided the conservation conditions are satisfied. The reflection probability of an excitation at the walls of the container is taken to be zero in the simulation. (In a separate experiment [10] employing a a large rotatable surface next to a source, we have shown that the contribution of reflected rotons to the observed signal is quite small.)

The heater is assumed to produce excitations predominantly near the roton minimum since the temperature of the heater during a pulse remains below $1 \mathrm{~K}$. The results 
of a simulation with excitations limited to the range $1.85 \AA^{-1}<k<1.95 \AA^{-1}$ is shown in Fig. 7 (solid line) and is in excellent agreement with the data. For comparison a simulation with excitations generated uniformly in momentum space up to a cut-off of $2.3 \AA^{-1}$ is also shown (dashed line) and is considerably broader than the measurements. The different width of the two simulations is easy to understand. Since the critical angle is smallest for excitations close to the roton minimum, these excitations will lead to the narrowest position dependence.

The positional dependence of the signal from $\alpha$ particles stopped in the liquid is shown in Fig. 8. Since $\alpha$ particles generate a broad spectrum as discussed above, we have arbitrarily chosen a distinctive feature of the distribution, namely, the high energy shoulder, to characterize the positional dependence. The two curves are, as before, simulations assuming rotons either with momenta restricted to the minimum in the dispersion curve or uniformly distributed over the entire spectrum. For $\alpha$ particles, as well as for the heater, the comparison between the simulation and the data indicates that the excitations producing evaporation are primarily near the roton minimum.

The excitations produced by an $\alpha$ particle in the liquid are created primarily by secondary electrons generated in the ionization of He atoms by the $\alpha$. This is a nonthermal process, and the electrons have far greater energies $\left(>10^{3}\right)$ than the rotons they produce. Therefore the excitations should be created throughout the phononroton spectrum. At first sight it is surprising that our signal indicates only rotons near the minimum are present. At temperatures below $50 \mathrm{mK}$ where these observations were made, there are no thermal excitations (or ${ }^{3} \mathrm{He}$ impurities) in the liquid that could scatter the rotons and down-convert them to a thermal distribution near the minimum.

To understand this result we note that the track of a $5.5 \mathrm{MeV} \alpha$ particle is only $200 \mu \mathrm{m}$ in helium, and the average range of the secondary electrons is much shorter, on the order of $10 \mathrm{~nm}$. Thus, something in excess of $10^{9}$ excitations are created in a cylindrical volume of $10^{-13} \mathrm{~cm}^{3}$. Since the roton-roton scattering cross-section is the order of $10^{-14} \mathrm{~cm}^{2}$, a dense, strongly interacting gas of excitations is produced along the $\alpha$ track. Before propagating away from the $\alpha$ track the excitations will undergo mutual scattering, and thermalization within the initial, dense cloud is likely. This process will cause a narrowing of the initial distribution of excitations to a region in the vicinity of the minimum, which is consistent with our data.

It is possible from the measured size of the signal detected by the wafer to make an estimate of the product of the efficiency of generation of excitations by an $\alpha$ particle and the efficiency of evaporation. We use the simulation with thermalization of the rotons and compare the detected energy to the $\alpha$ energy. The comparison also must 
include parameters associated with helium atoms on the wafer surface such as sticking probability $(\sim 0.7)$ and binding energy $(\sim 100 \mathrm{~K})[12]$. We can conclude that as a lower bound the probability is 0.3 that a roton, incident on the surface at less than the critical angle, will evaporate a helium atom. This lower bound uses the limiting assumption that all of the energy of the $\alpha$ is converted into elementary excitations of the liquid.

We now return to the statement that the size of the detected signal may depend on the orientation of the $\alpha$ particle track. The rotons are created as a dense, interacting cylindrical cloud. They thermalize before propagating away. Simple geometrical considerations suggest that the number propagating away from the region of interaction in a direction perpendicular to the axis of the cylinder will be greater than the number propagating parallel to the axis. Instead of a "fireball" we have a "fire-cylinder" from which thermal radiation is anisotropic.

Further research, both analytical and experimental, must be performed to determine if this picture is correct. If such an dependence on track orientation carries over to the case of electrons, the implications for a detector of solar neutrinos are substantial. This is discussed in the accompanying renewal proposal. 


\section{BACKGROUND STUDY FOR A HELIUM DETECTOR}

We have completed our first detailed studies of potential backgrounds for a full scale (ten ton) superfluid helium detector. These studies have two components: a) Monte Carlo simulations of radioactive backgrounds to any apparent signal and the nature of potential cuts to reduce them and b) consideration of niaterials to be used as absorbers/shielding from potential sources of radioactivity and the purity of those materials themselves.

If we assume that the detector would be sited in an underground venue comparable in the depth and environment to the Gran Sasso laboratory, then the broad outlines of the background problems are as follows. With a target weight of only ten tons, the area and mass presented by the detector itself to the residual penetrating muons is small. The background sources are principally of two sorts. They arise from a) the laboratory walls and its contents exterior to the detector itself (e.g., fast and slow neutrons from fission products and $(\alpha, n)$ reactions from heavy elements and from muon interactions at depth; $\gamma$ rays from neutron capture, muon interactions or radioactivity) and b) materials used to contain/construct the neutrino detecting material itself (e.g., $\gamma$ rays from residual radioactive isotopes cosmogenically induced prior to being placed underground; $\gamma$ 's from neutrons captured in the detector construction materials). The detector target itself, being liquid helium, has no activity and of course that is one of its essential, major attributes for this application. This property is not possessed by any other material.

Gamma rays, because they predominantly produce Compton recoil electrons in helium, can give energy depositions which individually are indistinguishable from a solar neutrino event. Hence, they are the particles (and their sources) which must be most severely controlled and understood. Largely because of this, the strategy we have adopted is to shield the detector in a way that limits the background radiation, which does succeed in making energy deposition in the sensitive volume of the target, to that due to the target container(the cryostat) alone. There are now quite reliable measurements of neutron and $\gamma$ ray backgrounds encountered in existing sites such as the Sudbury mine and Gran Sasso [15]. Typical fluxes at Gran Sasso are $4 \times 10^{-6} \mathrm{~cm}^{-2}$ $\mathrm{sec}^{-1}$ for neutrons and $0.1 \mathrm{~cm}^{-2} \mathrm{sec}^{-1}$ for $\gamma^{\prime}$ ' at the cave surface. It has been shown [16] in detail in Monte Carlo calculations by the groups proposing the SNO and Borexino detectors what effect of placing several meters of water between the laboratory walls and the detector itself is on neutron and $\gamma$ attenuation. For example, the Borexino group has shown that $<1$ per day each of neutrons and $\gamma$ 's reach the outer surface of their 
last absorber shield. We use their calculations of that effect as a guide since our initial calculations are cousistent with their result but are of much lower statistical significance. One can do better than shown, however, by reducing the fraction of the moderated ncutrons which capture on the hydrogen (2.2 $\mathrm{MeV}$ gamma resulting) in the water by adding solutes low in heavy element content but which have other desirable properties such as large thermal neutron cross sections. The large thermal neutron capture cross section of boron $\left({ }^{10} \mathrm{~B} ; 3800\right.$ barn $)$, its relatively high natural abundance $(20 \%)$, its lack of direct branching to $\gamma$ emission, the low $\gamma$ energies $(0.48 \mathrm{MeV})$ from daughters, and the high solubility $(6.3 \mathrm{~g} / 100 \mathrm{~g}$ water $@ 30 \mathrm{C})$ of boric acid at room temperature suggest that a saturated solution of boric acid in water would be an inexpensive and very satisfactory outer shield. The hydrogen in the water serves as the thermalizer and the boron as the dominant neutron absorber. Boron gives an improvement of more than 3 orders of magnitude in the capture rate. Since copper itself (the most likely cryostat material) has a large thermal neutron absorption cross section ( $3.8 \mathrm{~b}$ with subsequent. $\gamma$ emission $<1.5 \mathrm{MeV})$ it is important to reduce the neutrons incident on the cryostat from the exterior to a negligible amount.

In Fig. 10 below is reproduced a high statistics study by the Borexino group of the effect of water without solutes (they need pure water for transmission of light to phototubes). It provides a bench mark for us. The figure as reproduced (and annotated by us) shows that after 1 meter of sulphucrete and 2.5 meters of water the neutrons are reduced by more than ten orders of magnitiude. Although we will carry out high statistics runs of our own on this at a later date, it seems reasonably certain that after 2.5 meters of boric acid aqueous solution and 1 meter sulphucrete the number of neutrons incident on the cryostat will be negligble ( $<0.1$ neutron/day). There will be a small but somewhat larger flux of low energy $\gamma$ 's remaining, however, and their further attenuation is discussed below in connection with that of the cosmogenic contamination of the cryostat itself.

Radiation from the silicon wafers themselves will not be a significant background into the helium. For example, if there were the order of 1500 wafers they would ronstitute roughly 2 kilograms of silicon. The beta decays of the principal contaminants (tritium and ${ }^{22} \mathrm{Na}$ [17]) occur only in a single wafer and do not cause a coincidence. The $1.275 \mathrm{MeV} \gamma$ from ${ }^{22} \mathrm{Na}$ occurs at a rate of only 0.32 counts $/ \mathrm{day} / \mathrm{kg}$ and so is negligible.

Copper is in many ways an ideal material for the cryostat not only for its excellent thermal and structural properties but also because it has no long lived cosmogenicaly generated isotopes of its own, and those of other elements are well measured and understood from double beta decay experiments [18]. The dominant cosmogenic isotopes 
are ${ }^{54} \mathrm{Mn}\left(0.835 \mathrm{MeV} \gamma, 512\right.$ days), ${ }^{58} \mathrm{Co}\left(0.81 \mathrm{MeV} \gamma, 70.8\right.$ days), and ${ }^{60} \mathrm{Co}(1.17$ and $1.33 \mathrm{MeV} \gamma$ 's, 5.27 years). Assuming the copper could be stored underground for one year prior to operation then the total equilibrium rate from the isotopes would be 0.02 decays $/ \mathrm{min} / \mathrm{kg}$. It is clear that the cryostat is likely to be the dominant contributor to "neutrino-like" background. We have performed a detailed Monte Carlo study of the effecton signal to noise from these sources.

Wc have used the GEANT Monte Carlo program from CERN (suitably modified to account for very low energy deposition) as the principal vehicle for these calculations. We have used as a model detector a cube of helium contained in a copper cryostat the outer dimensions of which were 5.6 meters on an edge. Typically, a fiducial volume of 4.0 meters on an edge was defined for signal cuts ( $\checkmark 22$ neutrino events/day for the full p-p and ${ }^{7} \mathrm{Be}$ flux). The region between the cryostat and fiducial volume was then filled with varying amounts of active helium and inactive moderator; the moderator served two purposes: to absorb/attenuate some of the $\gamma$ flux and to shift the spectrum in such a way as to increase the probability of multiple interactions in the heli $\mathrm{mm}$ of the emerging $\gamma$ 's.

The helium and most of the moderator materials considered in the calculations were of light nuclei and the $\gamma$ interactions are overwhelmingly Compton scatters with little or no photoelectric or pair production occurring ( $<0.03 \%$ in helium). Calculations were performed for two classes of moderator materials in varying thicknesses. These were polyethylene and several cryogenic gases (including nitrogen, carbon dioxide, argon and neon).

Carbon dioxide has many qualities which commend its use over other materials. Both carbo. and oxygen have low $(n, \gamma)$ cross sections -0.19 and $3.5 \mathrm{mb}$, respectively. Neither have any $\gamma$ emitting states with lifetimes greater than $70 \mathrm{sec}$. Carbon-14, of course, has a beta decay with an end point of $156 \mathrm{keV}$ but its extremely low abundance(< $\left.1 \times 10^{-14}\right)$, short range and low probability of radiating bremstrahlung $(0.1 \%)$ prevent it from being a problem. As with the other gases, $\mathrm{CO}_{2}$ will have a low content of heavy elements since it enters the cryostat in gaseous form prior to freezing on the walls.

For purposes of illustration, it is useful to look at the kind of features for signal-tonoise which result from the use of $50 \mathrm{~cm}$ of carbon dioxide lining the copper cryostat and the helium volume outlined above. In Fig. 11 is shown a LEGO plot of a single typical $300 \mathrm{keV}$ baskground $\gamma$ ray event; it is typical in that the median number of Comptons per event for the observed spectrum is 5 and that they have a mean length for the "shower" of about $40 \mathrm{~cm}$ in helium. A neutrino signal event will, of course, consist of a single recoil electron with a range less than $1 \mathrm{~cm}$. This suggests an algorithm for cuts 
to enhance signal-to-noise which we have implemented as follows: a) only one visible deposition per event, b) conversion point of deposition $<200 \mathrm{~cm}$ radial distance from center of fiducial volume, c) energy of deposition $<800 \mathrm{keV}$. In Fig. 12 is a histogram in which the results of a simulated 10 day run are plotted - 260 neutrino events from p-p (black) and ${ }^{7} \mathrm{Be}$ (hatch) as well as 373 background gamma events (white). This first cut gives a signal-to-noise ratio of 0.7 . However, because the mean free path for the $\gamma$ spectrum is $59 \mathrm{~cm}$ and that for the neutrinos approaches infinity in helium, we can make a substraction of the position- dependent background from the uniformlydistributed neutrino signal. The observed distribution of 462 events in the live helium volume between $200 \mathrm{~cm}$ and $230 \mathrm{~cm}$ is extrapolated into the fiducial volume. The distribution of events with the singularity and energy cuts applied is plotted in Fig. 13 for a 10 day run. Performing the extrapolation and subtraction on a bin-by-bin basis produces the result of Fig. 14 and signal-to-noise of $0.9 \pm 10 \%$; for purposes here the residual background has been taken to be the size of the quadratically compounded errors of the subtraction.

If we take a $70 \%$ operational duty factor for the detector and the full $\mathrm{p}-\mathrm{p}$ and ${ }^{7} \mathrm{Be}$ neutrino fluxes of the "standard model", about 5000 signal events would be expected in a year, assuming the flux is entirely of electron-type neutrin ss. Should oscillations occur such that neutrinos having only neutral current interactions compose a fraction, $F$, of that flux, then the number of events would be less; $5000 \times(1-0.83 \times F)$. The neutral current cross section for those neutrinos being roughly $1 / 6$ of that for electron-type neutrinos.

These are very encouraging results. It continues to alpear that there are as yet no technical obstacles to a solar neutrino experiment of the type envisioned. As details are gathered from our other experiments, we shall continue to refine and make more specific our Monte Carlo studies of backgrourds. 


\section{References}

1. J. Bahcall and R. Ulrich, Rev. Mod. Phys, 60, 297 (1988); J. Bahcall and M. Pinsoneault, Rev. Mod. Phys. 64, 885 (1992); S. Turck-Chieze, in Proceedings of $X V$ International Conference on Neutrino Physics and Astrophysics, Granada, Spain (1992), to be published (1993).

2. R. Davis et al,Proceedings of 21 st International Cosmic Ray Conference, Adeleide, Australia, edited by R. J. Protheroe, Univ. of Adelaide Press), Vol. 12, 143 (1990).

3. K. S. Hirata et al, Phys. Rev. Lett. 65, 1297 (1990); ibid. 65, 1301 (1990); ibid. 66, 9 (1991); Y. Suzuki et al, Proceedings of the International Symposium on Neutrino Astrophysics, Takayama, Japan (1992), to be published (1993).

4. P. Anselmann et al, Phys. Lett. B285, 376 (1992); ibid. B285, 390 (1992).

5. A. I. Abazov et al, Phys. Rev. Lett. 67, 3332 (1991); V. N. Gavrin in Proceedings of the XXVI International Conference on High Energy Physics, Dallas, Texas, edited by J. Sanford, AIP Press (1993).

6. J. Bahcall and H. Bethe, Phys. Rev. D47, 1298 (1993); S. Bludman et al, Phys. Rev. D47, 2220 (1993); V. Barger et al, Phys. Rev. Lett. 69, 3135 (1992); J. Bahcall and H. Bethe, Phys. Rev. Lett. 65, 2233 (1990).

7. R.E. Lanou, H. J. Maris and G. M. Seidel, Phys. Rev. Lett. 58, 2498 (1987); also, Brown Univ., Dept. of Phys. Report DOE/ER/40452-5.

8. E. E. Haller of Lawrence Berkeley Laboratory very generously aided us in this technology.

9. F. Probst, S. Cooper, D. Drummer, M. Frank, and W. Seidel in Low Temperature Detectors for Neutrinos and Dark Matter IV edited by N. E. Booth and G. L. Salmon, Editions Frontieres, Gif-sur-Yvette, France (1992), p.193. Also, private communication.

10. F. S. Porter, Ph.D Thesis, Brown University (1993), unpublished.

11. F. S. Porter, S. R. Bandler, C. Enss, R. E. Lanou, H. J. Maris, T. More, and G. M. Seidel, Proceedings of the 20th International Conference on Low Temperature Physics, Eugene, Oregon (1993), Physica (to be published).

12. F. S. Porter, S. R. Bandler, C. Enss, R. E. Lanou, H. J. Maris, T. More and G. M. Seidel,Proceedings of the 7th International Conference on Phonon Scattering, edited by R. Pohl, Springer-Verlag, in press.

13. C. Enss, S. R. Bandler, F. S. Porter, R. E. Lanou, H. J. Maris, T. More and G. M. Seidel, Proceedings of the 20th International Conference on Low Temperature Physics, Eugene, Oregon (1993), Physica (to be published). 
14. A. F. G. Wyatt, Physica 128B, 392 (1984).

15. Bellotti et al, INFN Raport 85-i9 (1885); P. Belli et al, N.C. $101 \mathrm{~A}, 959$ (1989); G. Ewan. et al, Sudbury Neutrino Observatory Proposal 87-12 (1887).

16. Borexino Proposal Vol.1, 185-215 (1991) and Sudbury Neutrino Observatory Report \# SN0-87-12 (1987).

17. J.Martoff, Science 237, 507 (1987).

18. F. Avignone, et al, Phys. Rev. C34, 666 (1986) and private communication F. Avignone, F. Boehm, D. Caldwell. 


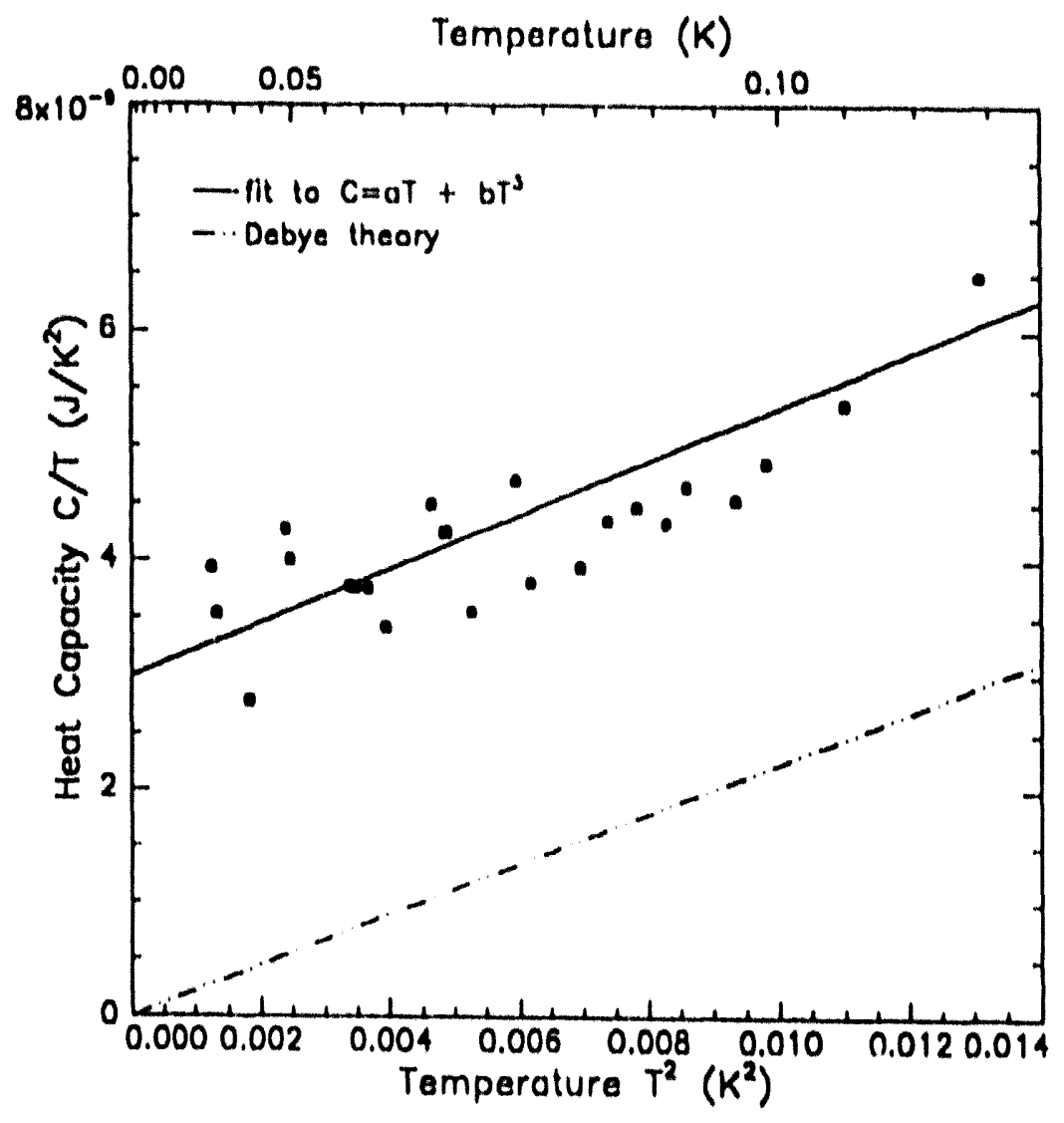

Figure 1. Heat capacity of a silicon wafer. $\mathrm{C} / \mathrm{T}$ versus $\mathrm{T}^{2}$. 


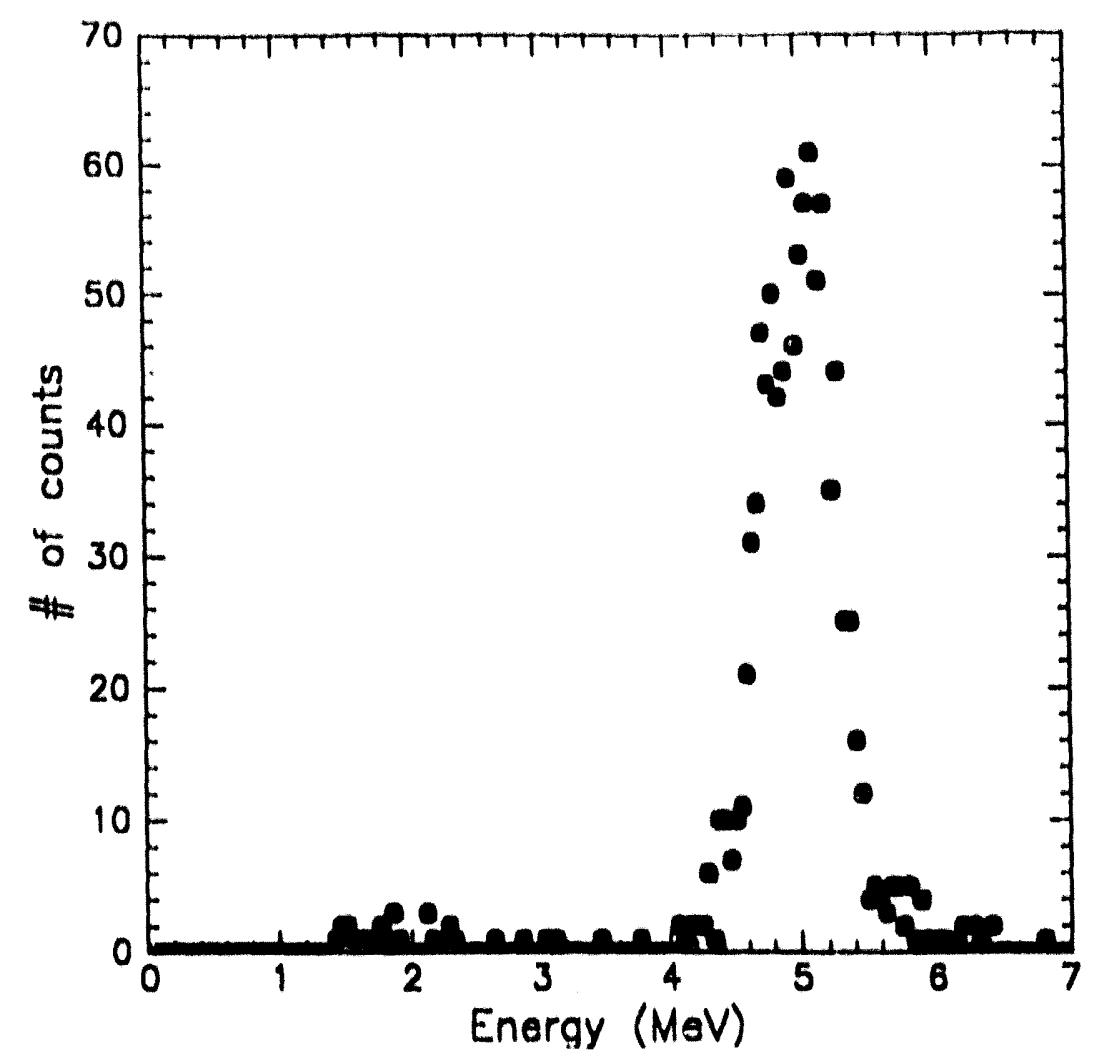

Figure 2. Pulse height distribution for $5.5 \mathrm{MeV} \alpha$ particles incident directly on a $20 \mathrm{~cm}^{2}$ wafer at $20 \mathrm{mK}$.

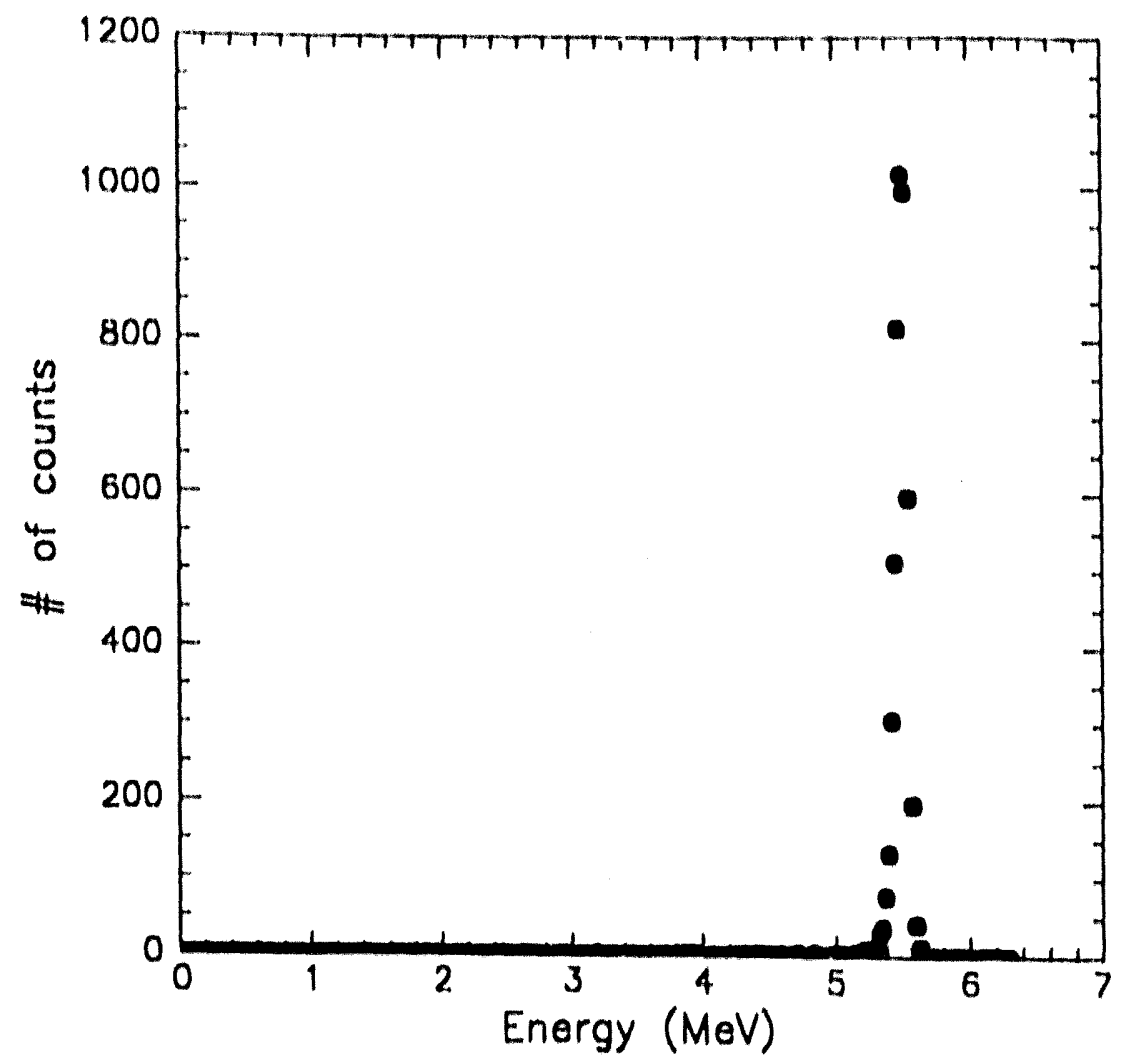

Figure 3. Pulse height distribution for a collimated beam of $5.5 \mathrm{MeV} \alpha$ particles incident a $0.2 \mathrm{~cm}^{2}$ area of a wafer at $20 \mathrm{mK}$. 


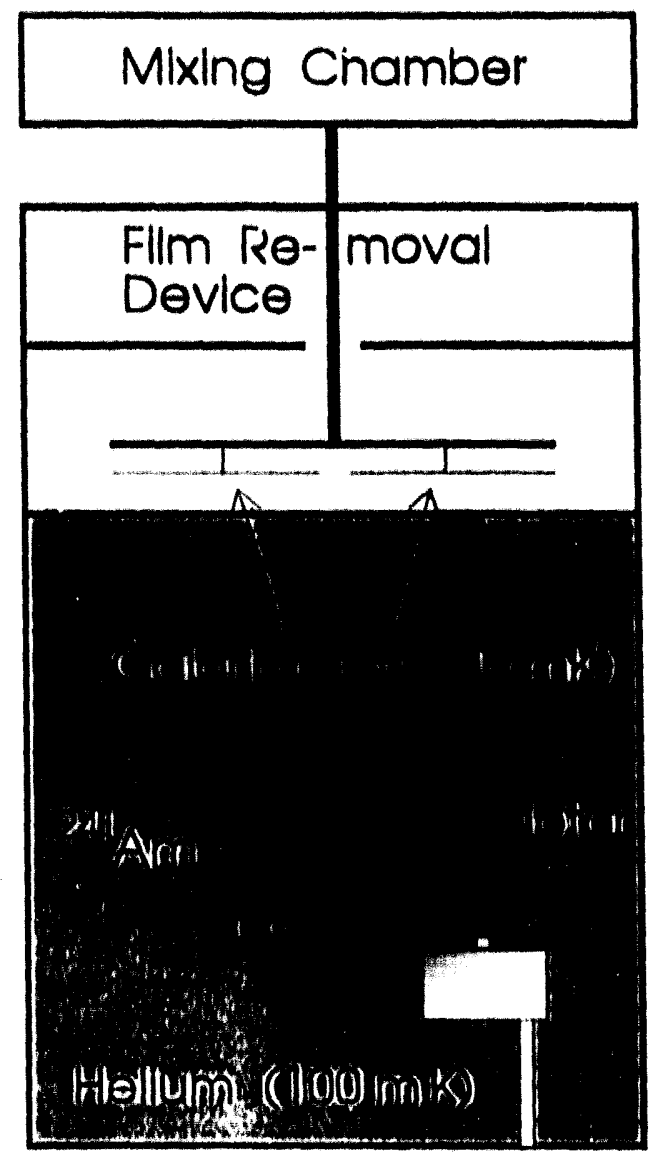

rigure 4. Schematic view of 3 liter cell with components. 


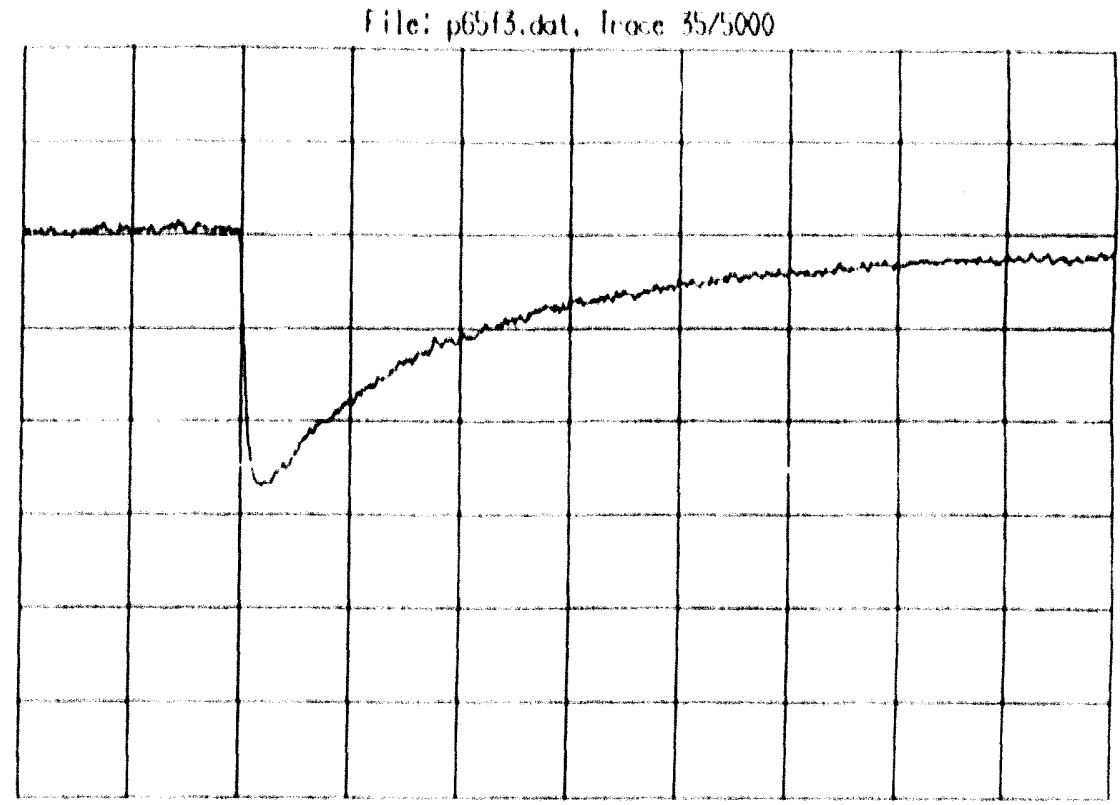

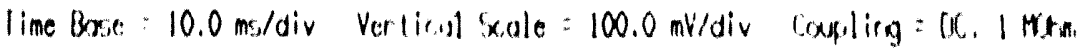

Figure 5. The thermal pulse on a sapphire wafer resulting from a E.5 Mev $\alpha$ particle stopped in helium, $5 \mathrm{~cm}$ below the liquid surface.

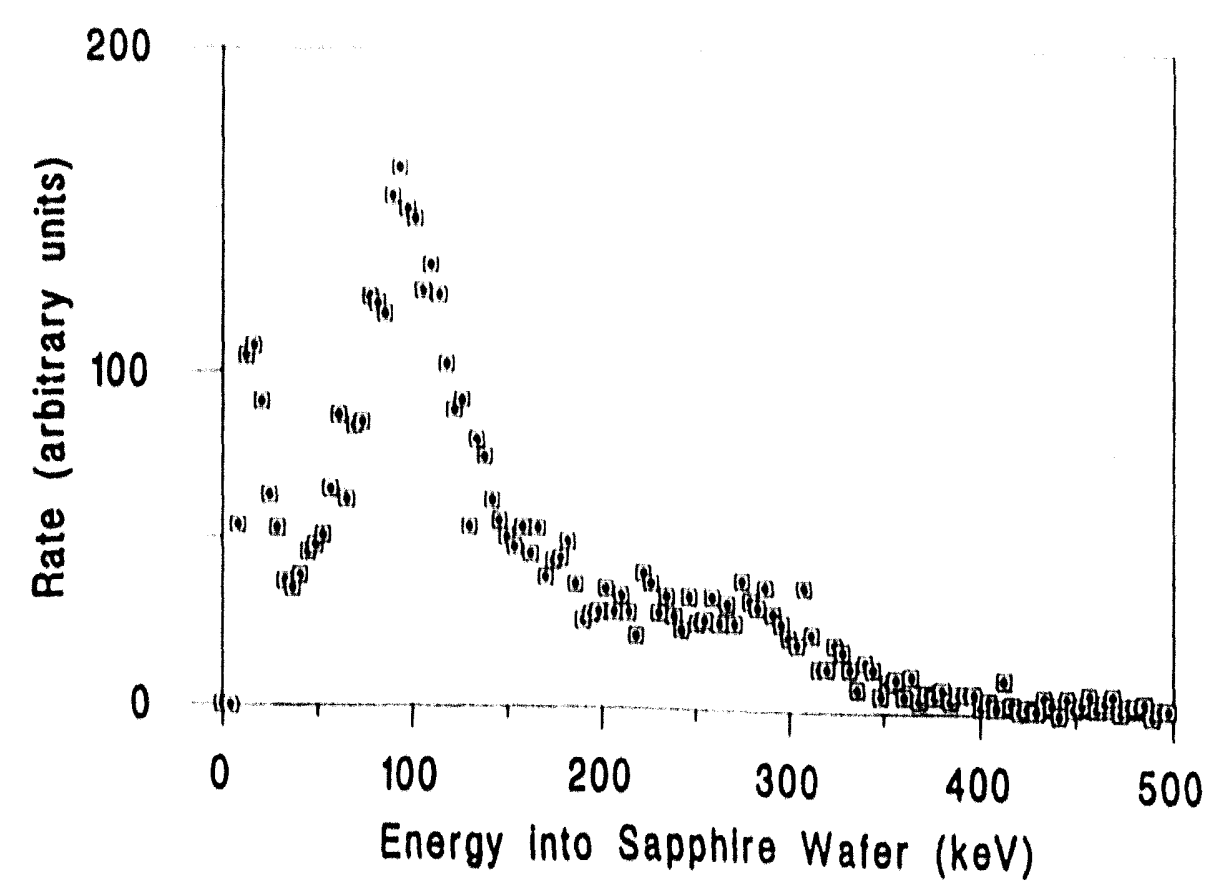

Figure 6. The pulse height spectrum of energy depositions into a sapphire wafer produced by $5.5 \mathrm{MeV} \alpha$ particles stopped in helium. The apparent low energy peak at $20 \mathrm{keV}$ is due to noise. 


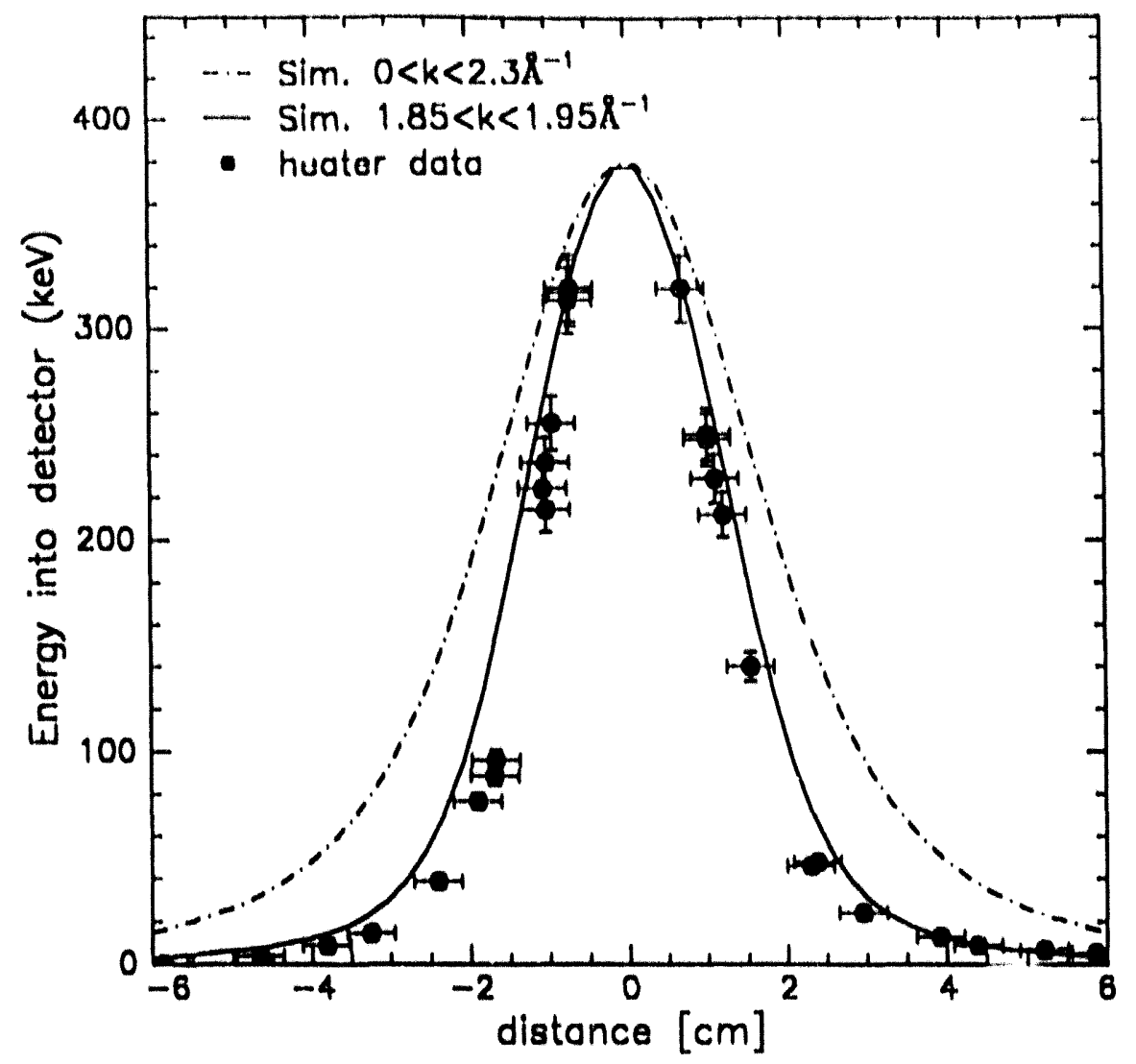

Figure 7. Evaporation signal as a function of the position of the pulsed heater. The maxima of the simulations are normalized to the maximum of the data.

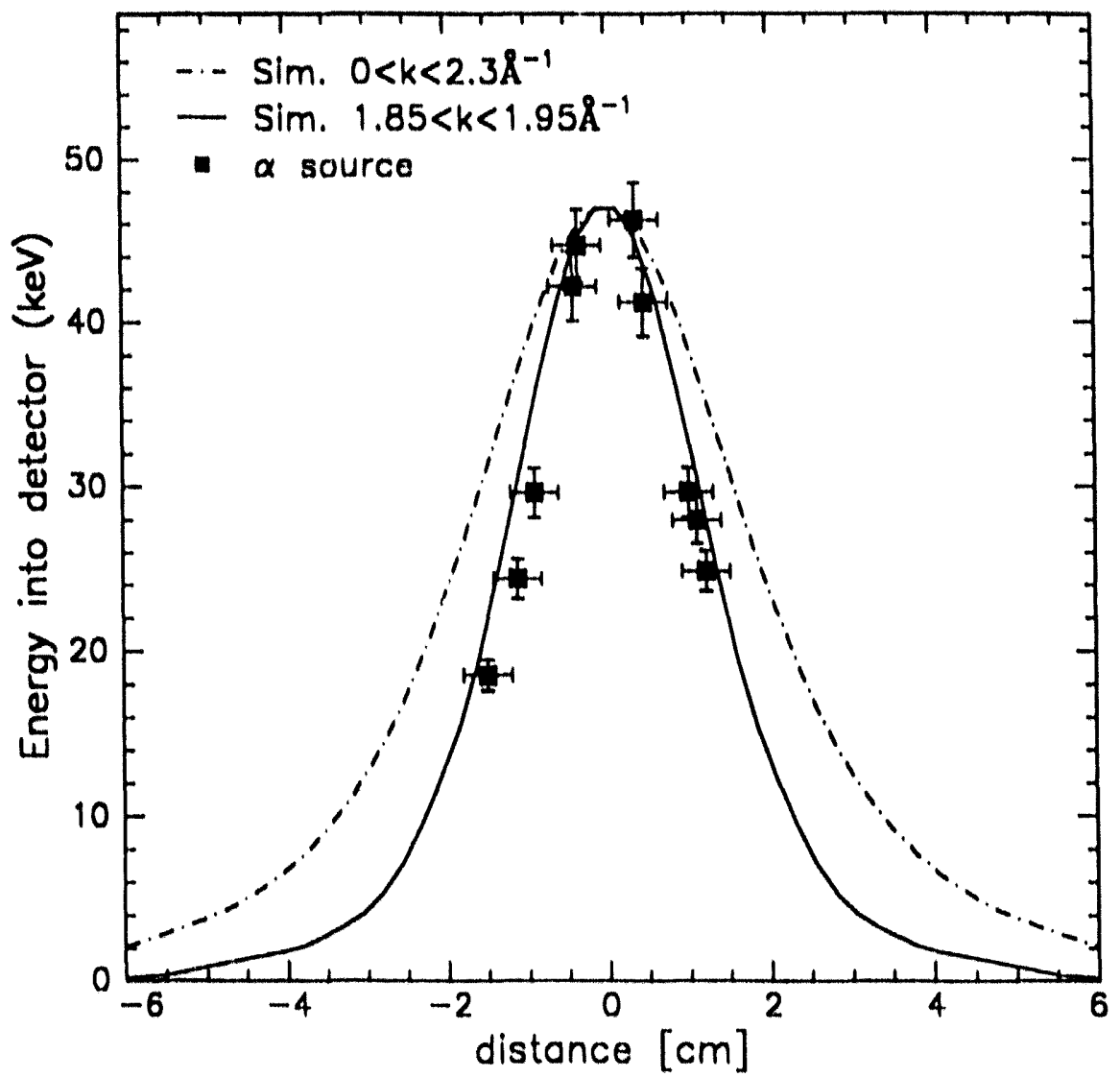

Figure 8. Evaporation signal as a function of the position of the $\alpha$ source. The maxima in the simulations are normalized to the maximum of the data. 

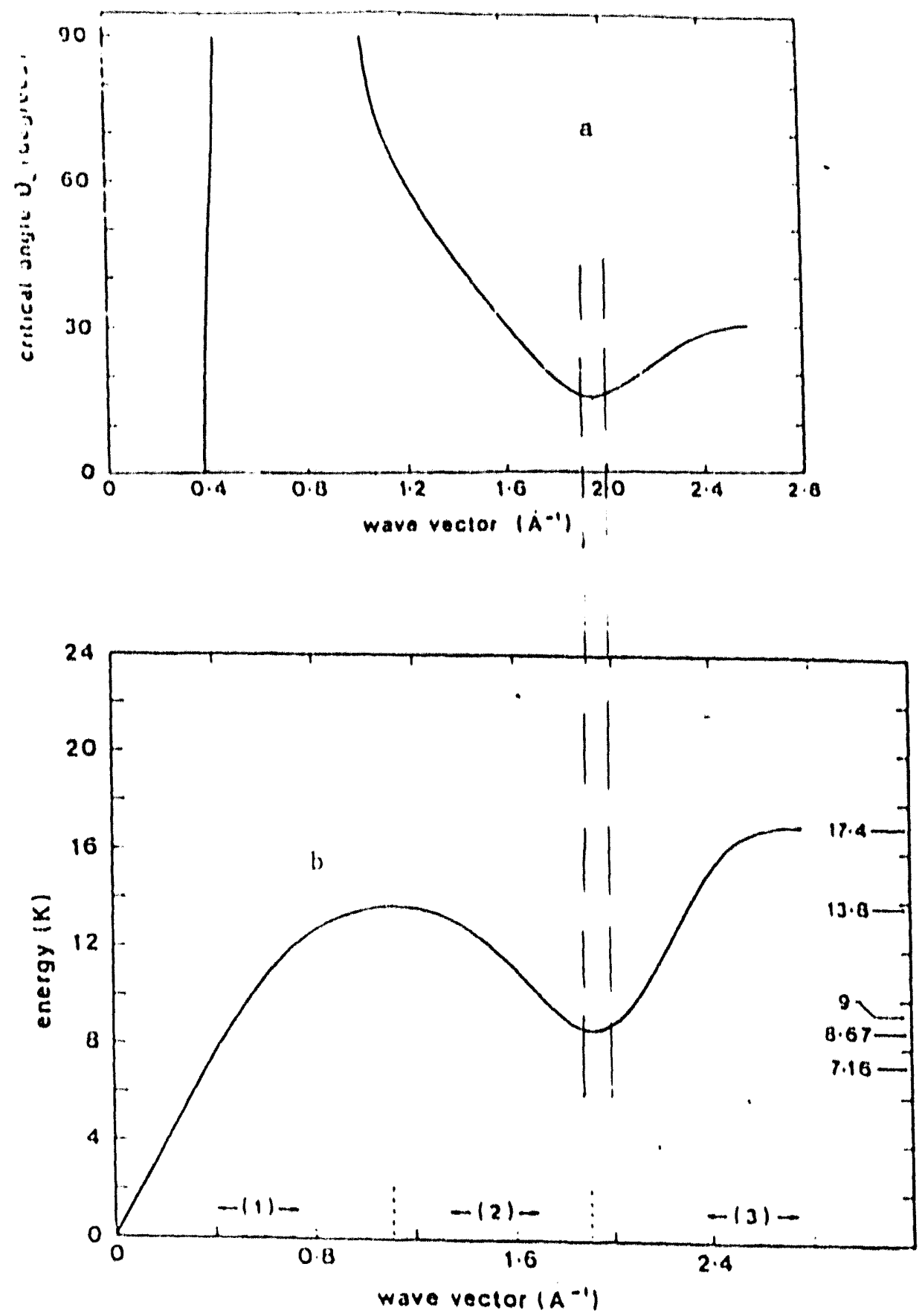

Figure 9.. a) Critical angle vs. momentum; b) Dispersion curve of excitntions in helium. Curves are taken from Ref. 14 and are plotted with same momentum scale. Vertical lines are added to show the momentum region best representing our data. 


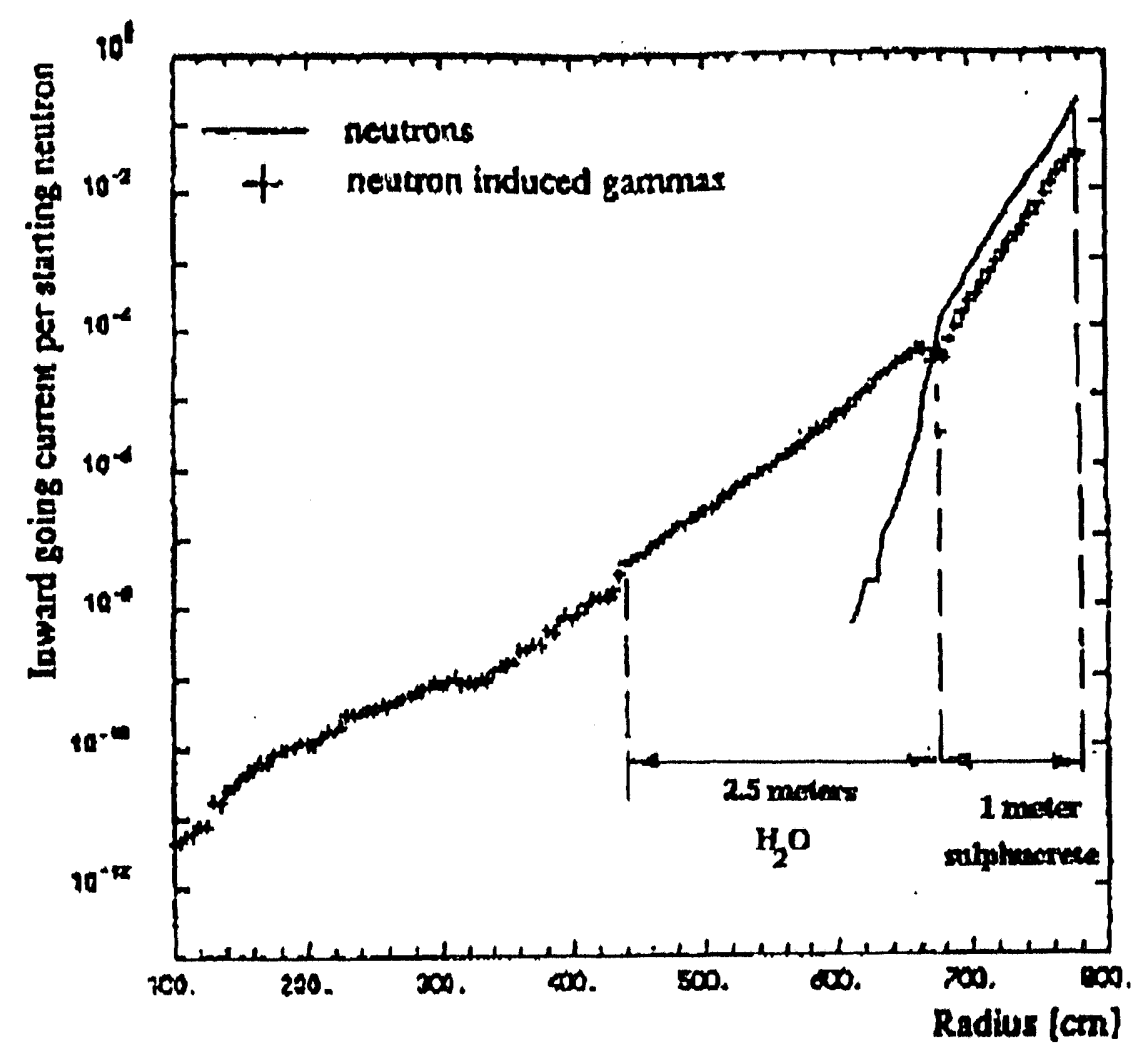

Figure 10. This figure is taken from the Borexino proposal with additional notation added for clarity.

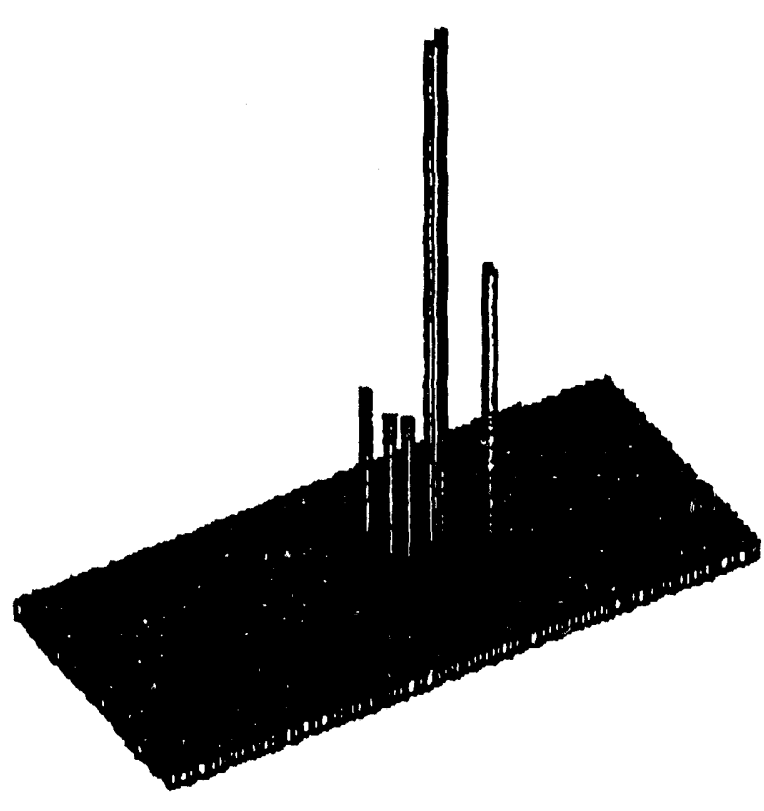

Figure 11. Horizontal distribution ( $x-y)$ of a $300 \mathrm{keV}$ gamı a ray interaction in liquid helium. Each "tower" is a single Compton scatter and its height is proportional to the recoiling electron energy. Each bin is $2 \times 2 \mathrm{~cm}$. The event is contained in an area $\sim 100 \times 60 \mathrm{~cm}$ (Monte Carlo). 


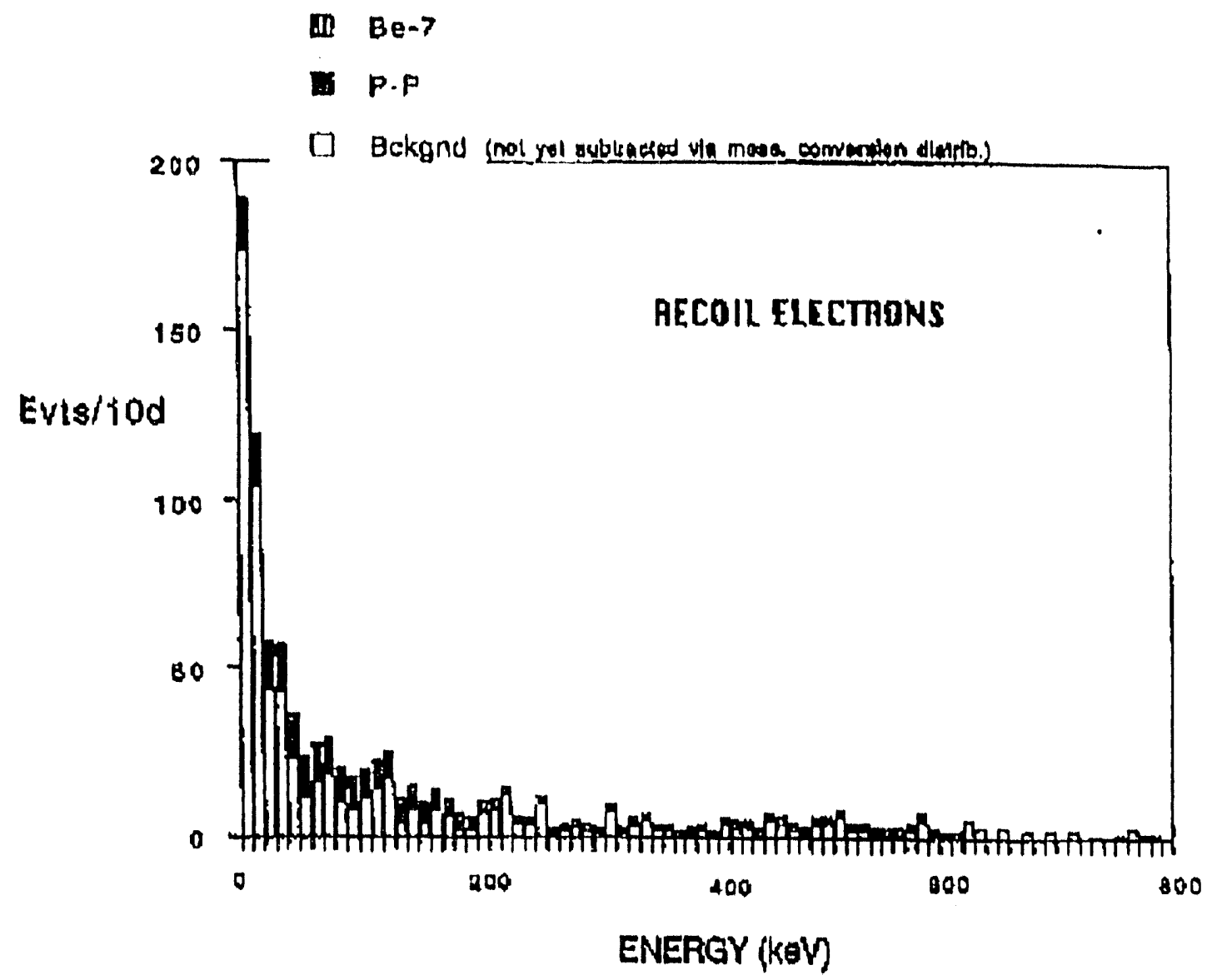

Figure 12. Monte Carlo result for 10 day run in 10 tons of liquid helium.

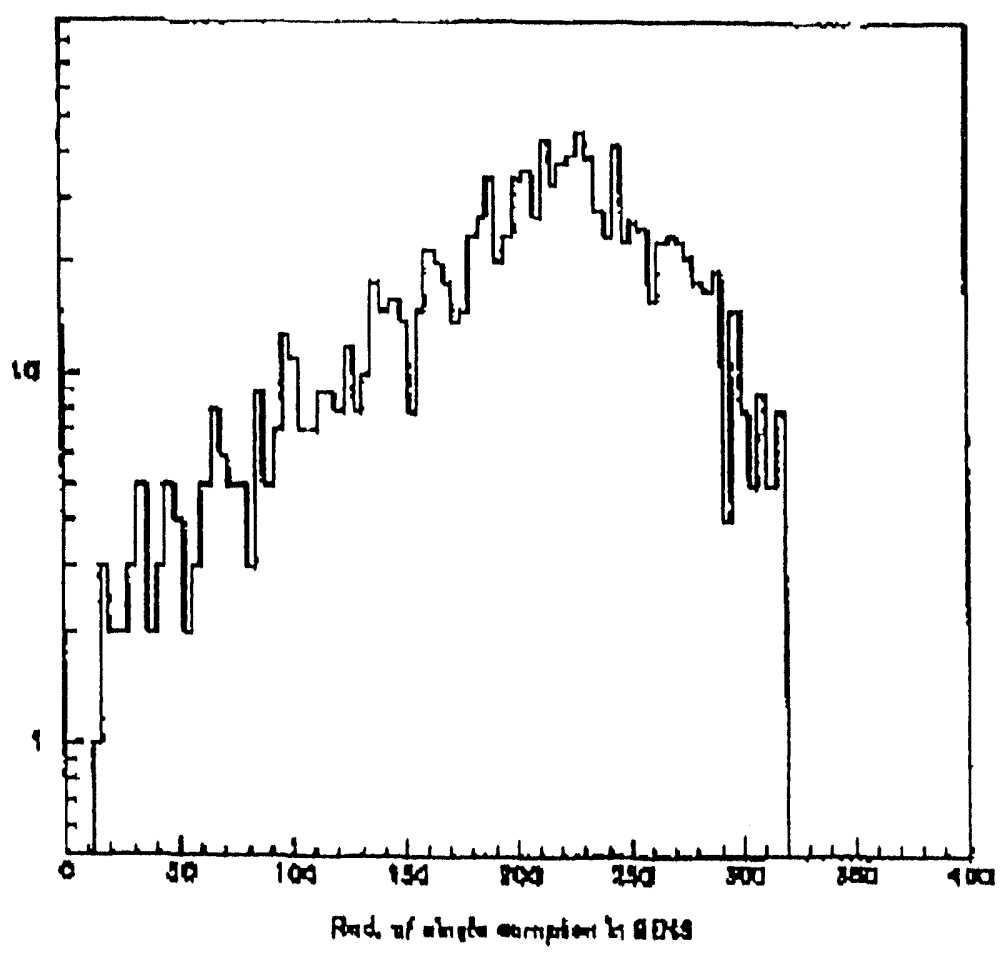

Figure 13. Radial distribution of Compton scatters in liquid helium. The events at sites larger than $230 \mathrm{~cm}$ arise because of the square nature of the cryostat in this Monte Carlo model. 


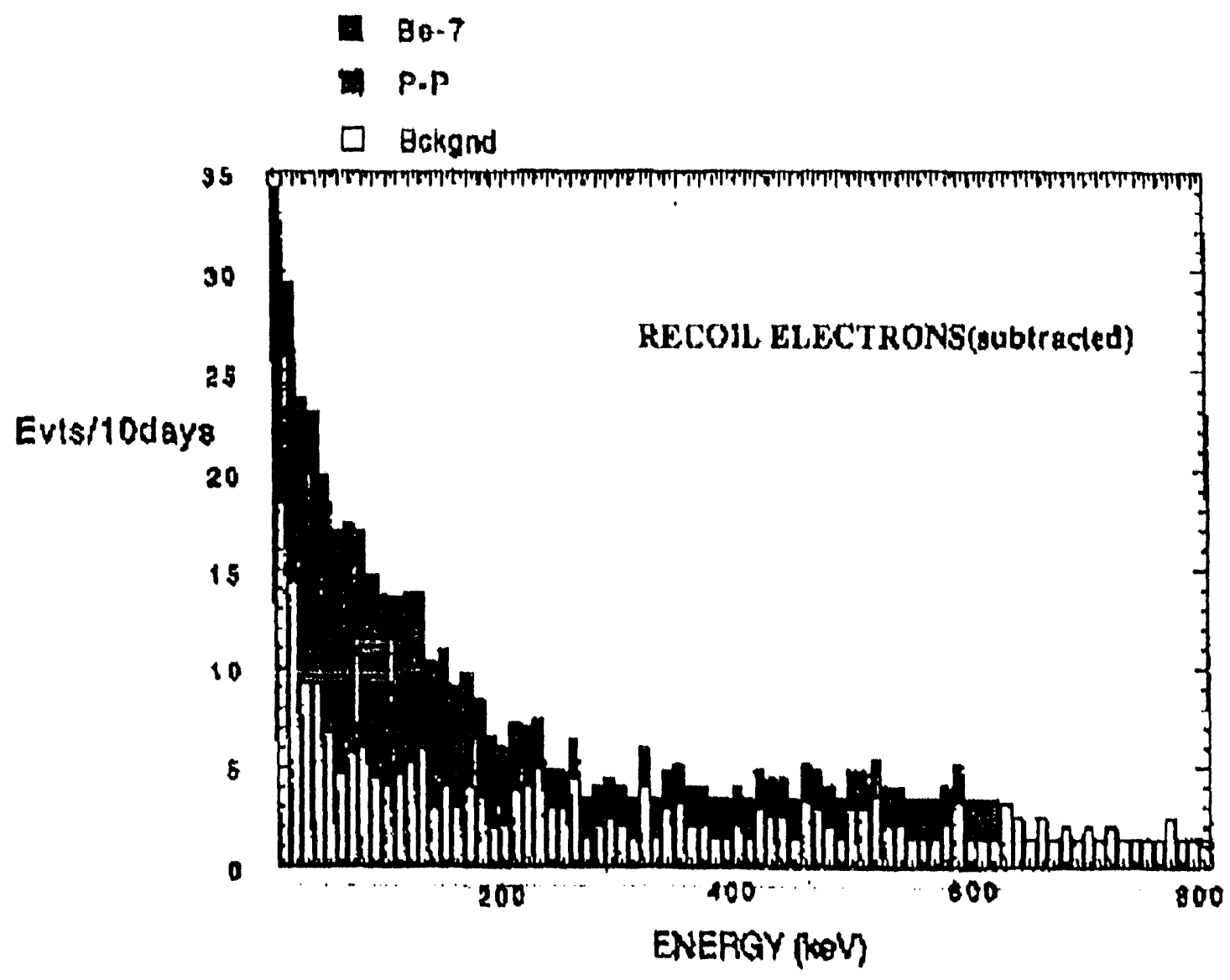

Figure 14. Monte Carlo of background subtracted 10 day run in 10 tons of liquid helium. The white bins are to indicate the error in the subtracted background. 


\section{RECENT PUBLICATIONS}

- S. R. Bandler, R. E. Lanou, H. J. Maris, T. More, F. S. Porter, G. M. Seidel, and R. Torii, "Particle Detection by Evaporation from Superfluid Helium", Phys. Rev. Lett. 68, 2429 (1992).

- F. S. Porter, S. R. Bandler, C. Enss, R. E. Lanou, H. J. Maris, T. More, and G. M. Seidel, "Calorimetric Detection of 5.5 MeV Alpha Particles in Superfluid Helium-4", Proceedings of 7 th International Conference on Phonon Scattering, edited by R. Pohl, Springer-Verlag, in press.

- F. S. Porter, S. R. Bandler, C. Enss, R. E. Lanou, H. J. Maris, T. More, and G. M. Seidel, "A Stepper Motor for Use at Temperatures Down to $20 \mathrm{mK}$ ", Proceedings of the 20th International Conference on Low Temperature Physics, Eugene, Oregon (1993), Physica (to be published).

- C. Enss, S. R. Bandler, F. S. Porter, R. E. Lanou, H. J. Maris, T. More and G. M. Seidel, "Quantum Evaporation of Helium-4: Angular Dependence and Efficiency", Proceedings of the 20th International Conference on Low Temperature Physics, Eugene, Oregon (1993), Physica (to be published).

- R. E. Lanou, H. J. Maris, and G. M. Seidel, Progress Report DOE/ER 140452-9 (1993).

\section{Other related matters:}

- The Ph.D. thesis of F.S. Porter, which derives from experimentaion performed under this grant, is in preparation and he is expected to receive his degree this Spring.

- S. R. Bandler and T. More will continue on their $\mathrm{Ph}$. D. research involving experiments to improve the sensitivity of calorimetric detection and to investigate the detection of electrons in helium. Their theses will be on subject of particle detection.

- At the 1993 Spring Meeting of the American Physical Society held in Washington, D.C. (April 12-15), an invited paper was presented on the results from this grant to a special Joint Session of the Division of Nuclear Physics and the Division of Fundamental Constants and Precision Measurements. Additional requests for seminars and colloquia on this novel technique and its goals have continued throughout the year. A feature article by the English physicist P. McClintock decribing this work appeared recently in Physics World, the journal equivalent to Physics Today in Great Britain. 


\section{SCIENTIFIC PERSONNEL}

$\begin{array}{ll}\text { R. E. Lanou } & \text { Professor of Physics } \\ \text { H. J. Maris } & \text { Professor of Physics } \\ \text { G. M. Seidel } & \text { Professor of Physics } \\ \text { G. Goldhaber* } & \text { Visiting Scholar (Research) } \\ \text { C. Enss** } & \text { Visiting Assistant Professor (Research) } \\ \text { F. S. Porter } & \text { Graduate Research Assistant } \\ \text { S. R. Bandler } & \text { Graduate Research Assistant } \\ \text { T. More } & \text { Graduate Research Assistant } \\ \text { J. Macbeth } & \text { Undergraduate Assistant in Physics }\end{array}$

* Professor Goldhaber is a nuclear/particle physicist visiting for the year from the University of California(Berkeley). He has been actively involved with the project but receives no funds from this grant.

** Dr. Enss is a low temperalure physicist from the University of Heidelberg. His principal support derives from the Alexander von Humboldt Foundation. 


\section{APPENDIX A. Principles of the Superfluid Helium Detector}

The limitations associated with the existing detection techniques for $\mathrm{p}-\mathrm{p}$ and $\mathrm{Be}$ solar neutrinos have led us to consider another using superfluid helium as a detector of neutrinos. Liquid helium can be used as the major component of a very sensitive calorimetric detector, with the major advantage of being able to achieve very low background levels. We discuss below the principles upon which such a detector is based.

From the point of view of the elimination of background, the ideal detector material is certainly liquid helium. Helium is the only element which remains a liquid down to absolute zero. Consequently all other elements freeze out on the walls of the container of the liquid. For example, a $\mathrm{H}_{2}$ molecule in liquid ${ }^{4} \mathrm{He}$ has an energy approximately 80 $\mathrm{K}$ greater than in solid hydrogen. Thus, the solubility of $\mathrm{H}_{2}$ in helium is proportional to $\exp (-80 / T)$, and is totally negligible below $1 \mathrm{~K}$. If by some process an impurity is introduced into the interior of the liquid, it moves very rapidly through the superfluid liquid and quickly adsorbs onto the container wall. Thus, in contrast to solids, helium may be considered to be a self-cleaning system.

The idea of our experiment is shown in the cartoon of Fig. A1. A solar neutrino of energy $E_{\nu}$ scatters elastically off an electron, and transfers energy

$$
E_{\text {rec }}=\frac{2 m_{e} c^{2} E_{\nu}^{2} \cos ^{2} \theta}{E_{\nu}^{2}\left(1-\cos ^{2} \theta\right)+2 m_{e} c^{2} E_{\nu}+\left(m_{e} c^{2}\right)^{2}},
$$

where $\theta$ is the propagation direction of the electron relative to the direction of the incident neutrino. The energy of the recoil electron is transferred to the liquid helium in a few $\mathrm{mm}$ by a number of processes, secondary ionizations, the generation of elementary excitations in the helium (phonons and rotons) and the creation of other entities such as metastable $\mathrm{He}_{2}$ molecules in excited states. Much of the energy that initially goes into producing positive ions and electrons is most likely to end up as rotons, when the $\mathrm{He}^{+}$ions form $\mathrm{He}_{2}^{+}$complexes and the electrons create bubbles [A.1]. Some significant fraction of the total energy appears ultimately on de-excitation and recombination as photons, principally in the ultraviolet, since liquid helium is known to function as an effective scintillator [A.2]. Nonetheless, another substantial fraction of the energy must appear finally as elementary excitations of liquid " $\mathrm{He}$, the dispersion curve for which is shown in Fig. A2a. Because of the large phase space associated with rotons, as seen in Fig. A2b, almost all this energy will be at large mornenta in rotons and high frequency phonons. While no accurate values are yet known of the division of the kinetic energy of an electron in liquid helium, we know from our measurements that the fraction $f$ of the energy appearing as rotons is close to 0.5 . 
In superfluid helium at $T=0 \mathrm{~K}$ a roton is a stable excitation because, for kinematic reasons, there are no decay modes. At finite temperatures there is a thermal distribution of phonons and rotons in the liquid, and a roton is scattered by collisions with these thermal excitations. However, at $0.1 \mathrm{~K}$ the density of the thermal excitations is so low that the rate of scattering of a roton is negligible [A.3]. Rotons can also scatter from ${ }^{3} \mathrm{He}$ impurities in the liquid, but it is easy to reduce the ${ }^{3} \mathrm{He}$ concentration to a level such that this scattering is unimportant.

At the free surface of the liquid, rotons can induce evaporation of helium atoms. This process was first studied experimentally by Wyatt et al [A.4]. Our own measurements confirm and extend the earlier results. The conclusion is that a roton (or a high energy phonon) incident on the liquid surface has a probability of roughly $1 / 3$ of inducing the evaporation of 1 helium atom, provided that the kinematic constaints of conservation of energy and momentum parallel to the surface are satisfied in the evaporation process. Rotons that produce evaporation are restricted roughly to lie within a cone making an angle of $20^{\circ}$ with the normal to the surface. Thus the ratio of evaporated helium atoms to rotons is only about $1 \times 10^{-2}$. This neglects all reflections from walls. For an electron recoil energy, $E_{\text {rec }}$ of $200 \mathrm{keV}$, the number of rotons produced is $\sim f E_{\text {rec }} / \Delta \sim 1.4 \times 10^{8}$. (We make the approximation that all rotons have an energy close to the minimum roton energy $\Delta=8.6 K$.) The number of helium atoms evaporated from the surface of the liquid is then $\sim 1 \times 10^{6}$.

To detect these ${ }^{4} \mathrm{He}$ atoms we suspend thin plates of a high purity, high Debye temperature, crystalline, dielectric material such as silicon $1 \mathrm{~cm}$ above the surface.

The ${ }^{4} \mathrm{He}$ atoms which evaporate will be adsorbed (physisorbed) onto the Si surface [A.5]. Each atom generates in the $\mathrm{Si}$ an amount of heat equal to the binding energy $\phi$ between ${ }^{4} \mathrm{He}$ and a Si surface [A.6]. This binding energy is roughly $100 \mathrm{~K}$, and originates in the van der Waals attraction between a helium atom and the silicon surface. Notice that the energy deposited in the $\mathrm{Si}$ is

$$
E_{S_{i}} \simeq 10^{-2} f \frac{\phi}{\Delta} E_{\text {rec }}
$$

Thus, with $f=0.5$, for a $200 \mathrm{keV}$ electron $E_{S i}$ is $2 \times 10^{-8} \mathrm{erg}$.

If the area of the Si plate is $\mathrm{A} \mathrm{cm}$ and the thickness if $\mathrm{d} \mathrm{cm}$, the heat capacity is

$$
C=6.25 \times 10^{-7} \text { Ad } T^{3} \text { joule } / \mathrm{K}
$$

As representative number we might take $A=100 \mathrm{~cm}^{2}, d=0.02 \mathrm{~cm}$ and $T=20 \mathrm{mK}$. Then the temperature rise $\delta T_{d}$ of the $\mathrm{Si}$ wafer caused by an electron recoil of $200 \mathrm{keV}$ is $0.2 \mathrm{mK}$, which is easily measureable. 
It is precisely this sequence of processes which we have been studying.

\section{References}

A.1 See A. L. Fetter in the Physics of Liquid and Solid Helium, edited by K. M. Benneman and J. Ketterson (John Wiley, New York 1976).

A.2 J. B. Birks, The Theory and Practice of Scintillation Counting (MacMillan, N. Y. 1964). M. Stockton et al, Phys. Rev. Lett. 24, 654 (1970); C. M. Surko et al, Phys. Rev. Lett. 24, 657 (1970).

A.3 S. Balibar et al, Phys. Rev. B18, 3096 (1978); H. J. Maris and R. Cline, Phys. Rev, B23, 3308 (1981).

A.4 M. J. Baird, F. R. Hope and A. F. G. Wyatt, Nature 304, 325 (1983); F. R. Hope, M. J. Baird and A. F. G. Wyatt, Phys. Rev. Lett. B2, 1528 (1984); for a review see A. F. G. Wyatt in PHONON89, edited by S. Hunklinger et al (World Scientific, 1989) p. 1019; G. Wyborn and A. F. G. Wyatt, Phys. Rev. Lett. 65, 345 (1990).

A.5 At $0.1 \mathrm{~K}$ the thermal equilibrium density of ${ }^{4} \mathrm{He}$ atoms above the surface is only $\sim 10^{-12} \mathrm{~cm}^{-3}$. Thus the rate at which these atoms hit the $\mathrm{Si}$ surface is negligible.

A.6 This binding depends upon the crystalographic orientation of the silicon surface. The figure of $100 \mathrm{~K}$ is an average value provided to us by M. J. Cardillo (A'TT-Bell Labs) based on a variety of experimental data. An oxide layer on the Si will change $\phi$ somewhat. See also R. Luchese and J. Tully, Surf. Sci. 137, 570 (1984). Our own measurements support the value of $100 \mathrm{~K}$. 


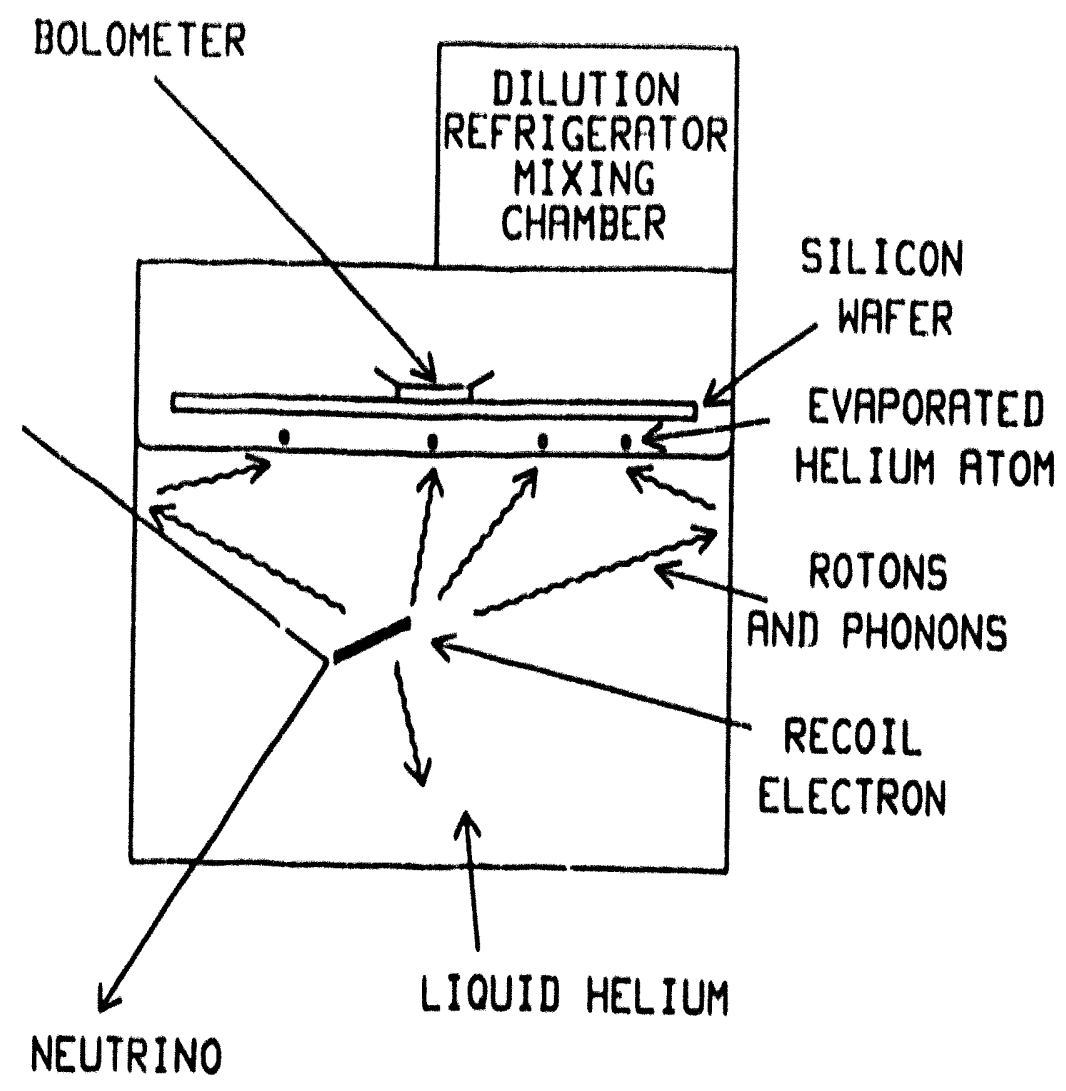

Figure 11 . Schematic diagram of the simplest version of the experiment. A neutrino is elastically scattered in the liquid helium. The recoil electron produces rotone and phonons in the liquid. When the rotons reach the surface, they evaporate helium atoms, which are then collected on the silicon wafer. The rise in temperature of the silicon is measured with a bolometer. Support for the silicon wafer is not shown.

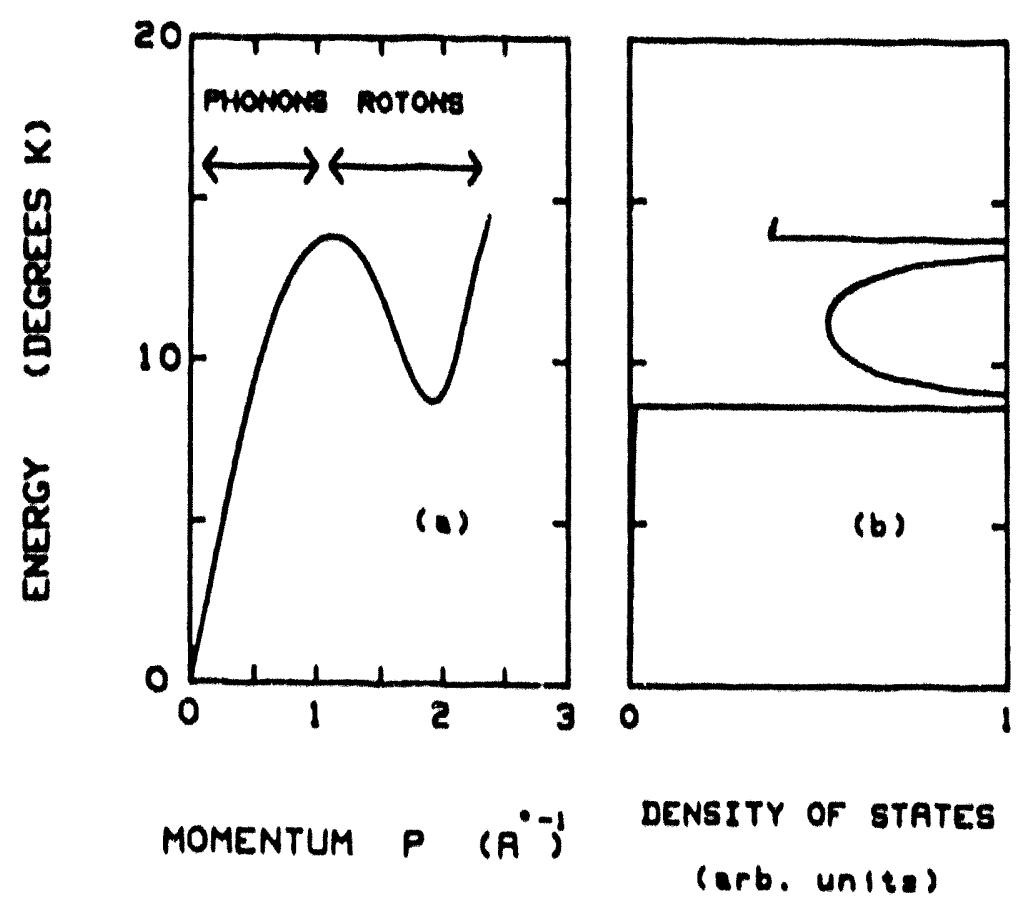

Figure $12 a$. Dispersion curve for elementary excitations in superfluid helium. Excitalions are divided into phonons (momentum less than $1 \dot{A}^{-1}$ ) and rotons $\left(2.4 \dot{A}^{-1}>p>\right.$
$\left.1 \dot{A}^{-1}\right)$.

Figure A2b. Density of states of excitations in arbitrary units. 

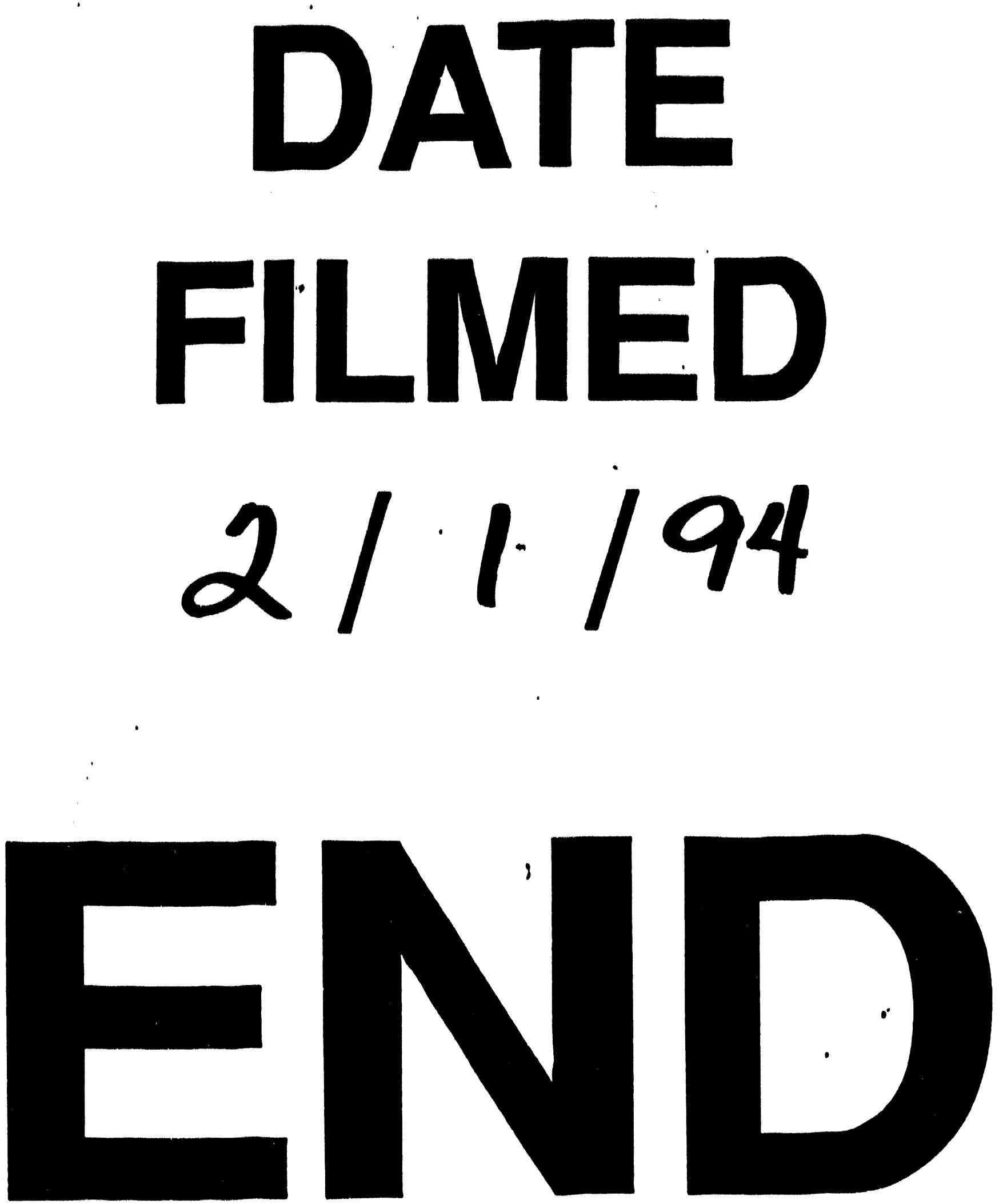
